\title{
Arrhythmias in Children and Young Adults
}

\author{
Harinder R. Singh \\ Assistant Professor, Pediatrics, Pediatric Electrophysiologist and Associate, Cardiology, \\ The Carman and Ann Adams Department of Pediatrics, Children's Hospital of Michigan, \\ USA
}

\section{Introduction}

One of the challenges that physicians taking care of children face is the interpretation of an electrocardiogram (ECG). The findings on ECG should be seen in the light of a patient's clinical presentation. In this evidence-based chapter, we will explore the most common pediatric arrhythmias, including sinus arrhythmia, premature beats, brady-arrhythmias, escape rhythms and tachy-arrhythmias including different types of atrial, junctional and ventricular tachycardias. The objective of this chapter is to familiarize the reader with ECG interpretation of common pediatric arrhythmias in children and young adults.

\section{Sinus arrhythmia}

2.1 Definition: Sinus arrhythmia is the normal variation in the rate of the sino-atrial (SA) node that is mainly associated with the respiratory phases.

2.2 Incidence: It is common in children and the incidence decreases with age(Kaushal and Taylor 2002).

2.3 Mechanism: It occurs due to change in sinus node function related to changes in the autonomic tone. Inspiration and expiration are associated with increased and decreased heart rate due to decreased and increased parasympathetic tone, respectively(Coker et al. 1984). Sinus arrhythmia disappears with conditions associated with elevated sympathetic tone causing increased heart rate.

2.4 Clinical presentation: Auscultation reveals irregular heart rhythm which varies with respiration.

2.5 ECG: ECG reveals sinus rhythm with variation in P-P interval preceding the change in R$\mathrm{R}$ intervals without any significant change in P-R interval (Figure 1).

2.6 Management: No work-up is needed because sinus arrhythmia is a normal variant with excellent prognosis.

\section{Ectopic complexes (premature beats)}

\subsection{Premature atrial contractions}

3.1.1 Definition: Premature atrial contraction (PAC), or atrial premature beat, is a premature discharge arising from an atrial focus other than the sino-atrial (SA) node.

3.1.2 Incidence: PACs were reported in 14\% of full-term infants on 24-hour ECGs (Southall et al. 1980). Among 10-13 year old boys with healthy hearts, $13 \%$ had singlet PACs (Scott et al. 


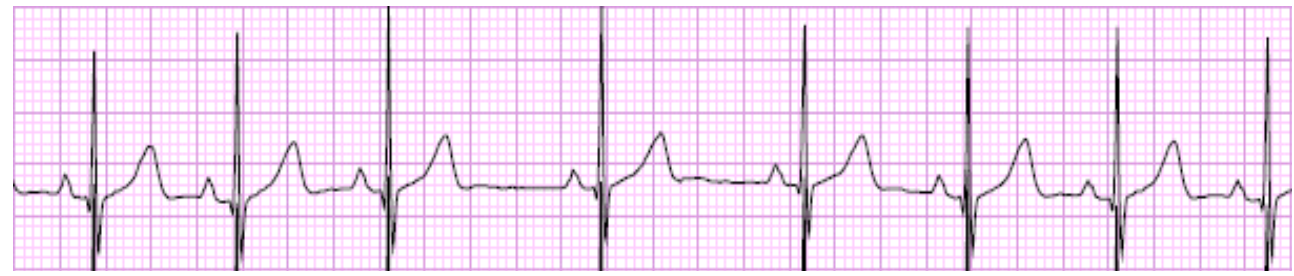

Fig. 1. Sinus arrhythmia

1980). On 24-hour ECG monitoring, 64\% of young women (Sobotka et al. 1981) and 56\% of male adolescents had PACs (Brodsky et al. 1977). Frequent causes of PACs include intake of caffeine, nicotine, alcohol, and other stimulants. PACs can be associated with atrial enlargement, electrolyte abnormalities, mechanical trauma, chronic pulmonary disease, and heart failure.

3.1.3 Mechanism: PACs can occur either due to a re-entry mechanism, (Wallace and Daggett 1964) automaticity outside of the SA node, or triggered activity (Wit and Cranefield 1977).

3.1.4 Natural history: PACs can give rise to atrial fibrillation and flutter, especially in older post-operative patients(Jideus et al. 2006). They may subside with removal or correction of the inciting stimuli. Control of congestive heart failure, ischemic heart disease, pulmonary disease, and electrolyte abnormalities may decrease the incidence of PACs.

3.1.5 Clinical presentation: Patients with PACs may be asymptomatic or perceive them as palpitations. They may be incidentally detected as irregular heart rhythm on auscultation.

3.1.6 ECG: A PAC presents as a P-wave that appears earlier than expected on an ECG. The P-wave morphology may appear different. The PR interval in a PAC is often different than the previous PR interval. PACs can have three possible outcomes: 1) block at the AV node due to AV nodal refractoriness (Figure 2a), 2) conduct normally through the AV node (Figure 2b), or 3) conduct with aberrancy due to His-bundle refractoriness (Figure 2c). There is no compensatory ventricular pause.

3.1.7 Work-up: A 12-lead ECG is usually adequate for evaluation; however, Holter monitoring may be performed.

3.1.8 Management and Prognosis: Control of exogenous factors will decrease the incidence of PACs. No physical restrictions need to be imposed on children without heart and/or lung disease, or electrolyte abnormalities. Beta blocker like bisoprolol decreased PACs by 50\%, although $25 \%$ of these patients had adverse reactions associated with beta blockade (Sugimoto et al. 1986). Prognosis is excellent for patients with PACs with normal hearts and needs no treatment.

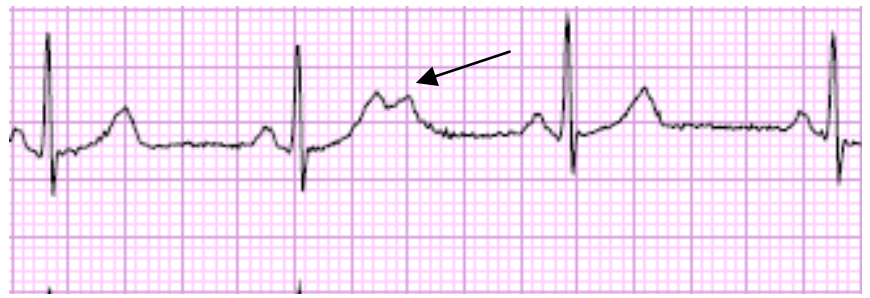

Fig. 2.a Premature atrial complex that is blocked 


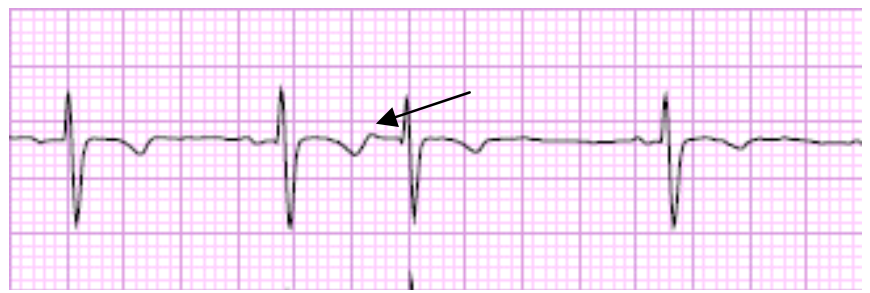

Fig. 2.b Premature atrial complex that is normally conducted

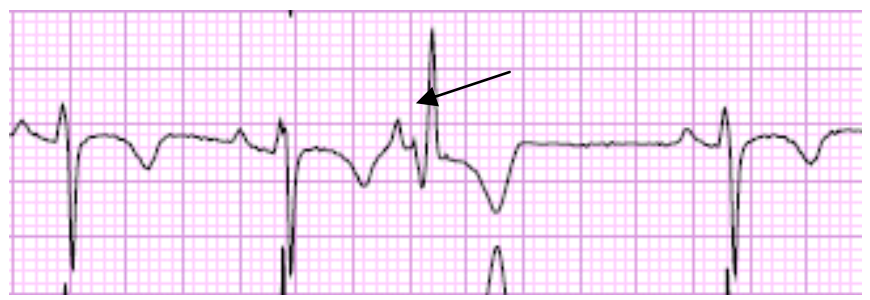

Fig. 2.c Premature atrial complex that is conducted with aberrancy

\subsection{Premature ventricular contractions}

3.2.1 Definition: A premature ventricular contraction (PVC) or ventricular premature beat (VPB) is an early ventricular beat that originates below the bifurcation of the bundle of His. It is characterized by a premature wide QRS complex that is morphologically different compared to the baseline QRS complexes (Sherron et al. 1985; Olgin and Zipes 2005b).

3.2.2 Incidence: PVCs follow a bi-modal distribution and are seen in $18 \%$ of neonates, $20 \%$ of toddlers, $16 \%$ of school children, $20-30 \%$ of adolescents and subsequent increases in late adulthood (Southall et al. 1980). On 24-hour ECG monitoring of subjects without heart disease, $54 \%$ of young women (Sobotka et al. 1981) and 50\% of young males had PVCs (Brodsky et al. 1977). Surgically-corrected Tetralogy of Fallot patients have an incidence of 648\% (Vaksmann et al. 1990),(Chandar et al. 1990).

3.2.3 Mechanism: Three mechanisms inducing PVCs include: automaticity, re-entry, and triggered activity. PVCs can result from mechanical, electrical, and chemical stimulation leading to ventricular depolarization. Abnormalities in metabolism, electrolytes, or iatrogenic causes can initiate PVCs in children. Both slow and fast heart rates are associated with PVCs (Sherron et al. 1985).

3.2.4 Natural history: PVCs increase with age and are more common in males (Silka and Garson 1999). In the absence of heart disease, PVCs are inconsequential under the age of 30 years but influence risk of sudden death in those over 30 years (Chiang et al. 1969). PVCs may be present in those with increased vagal tone such as athletes or in those with increased stimulation such as intake of caffeine, alcohol, and/or nicotine. In adults without structural heart disease, PVCs induced during exercise and recovery phases were associated with increased risk of mortality (Frolkis et al. 2003). PVCs also occur in those with surgicallycorrected congenital heart disease, myocarditis, right-ventricular dysfunction, congestive heart failure, cardiomyopathy, ventricular hypertrophy, myocardial infarction, ventricular non-compaction and iatrogenic causes (Chandar et al. 1990; Vaksmann et al. 1990; EspinolaZavaleta et al. 2006; Sestito et al. 2007). 
3.2.5 Clinical presentation: PVCs are the most common cause of irregular heart beats in children. They are usually detected incidentally and occasionally produce symptoms. Single PVCs are generally well-tolerated by children. Symptoms secondary to PVCs include palpitations, lightheadedness, fatigue, chest pain, and shortness of breath.

3.2.6 ECG: To be classified as PVC, four criteria should be met: 1) premature QRS without a premature $\mathrm{P}$ wave, 2) difference in QRS morphology between PVC and regular QRS complex, 3) prolonged QRS duration for age, and 4) different QRS and T wave vectors. Unifocal PVCs have a fixed morphology, as opposed to PACs with aberrant conduction. Multifocal PVCs have different morphologies due to the different locations of onset and electrical instability of the ventricle (Silka and Garson 1999). There is a compensatory pause after PVC, i.e., R-R interval produced by SA node initiated QRS complexes on either side of the PVC is equal to twice the normal R-R interval (figure 3). PVCs may present as isolated, couplets, bigeminy or trigeminy pattern. If the PVC originates in the right ventricle, the QRS morphology appears as a left bundle branch block pattern and if the PVC originates in the left ventricle, the QRS morphology appears as a right bundle branch block pattern.

3.2.7 Differential diagnoses: 1) Premature atrial complex with aberrant conduction, 2) premature junctional complex with aberrant conduction, and 3) anterograde conduction over an accessory pathway with pre-excitation.

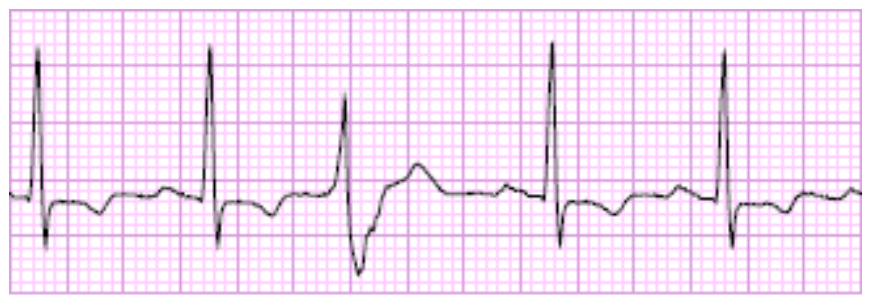

Fig. 3. Premature ventricular complex with a compensatory pause

3.2.8 Work-up: A detailed history of episodes is necessary. A history of congenital heart disease with subsequent surgical correction should be obtained. In addition to an ECG and 24-hour Holter monitoring, an exercise test is important in determining whether the frequency of PVCs or symptoms worsen with activity (Paridon 2006; Zipes 2006). Multifocal PVCs in structural heart disease require further workup. Echocardiography and electrophysiology studies may be warranted in surgically corrected patients or those with structural heart disease if there are PVCs and malignant ventricular arrhythmias (Case 1999).

3.2.9 Management and Prognosis: Management of PVCs depends on two factors: 1) a history of structural heart disease or surgically corrected congenital heart disease, and 2) the symptoms experienced by the patient. In children, PVCs are considered to be benign if they disappear with exercise (Attina et al. 1987). The majority of children with normal hearts presenting with asymptomatic single PVC, bigeminy, or trigeminy do not require treatment. Given that surgically-corrected congenital heart disease is associated with increased predisposition to non-sustained ventricular tachycardia and sudden cardiac death, children may require treatment with anti-arrhythmics under the supervision of a pediatric cardiologist. Beta-blockers can assist in suppressing PVCs in symptomatic patients (Alexander 2001a). If symptoms are not medically controlled, catheter ablation is an option (Zhu et al. 1995). Prognosis depends on whether there is underlying structural and 
metabolic heart disease. PVCs inducible during exercise can be ominous in patients with structural heart disease and are of higher significance (Silka and Garson 1999). Athletes with structural heart disease in high-risk groups with PVCs should be restricted to low-intensity sports (Zipes and Garson 1994).

\subsection{Premature junctional complexes}

3.3.1 Definition: A premature junctional complex (PJC) or beat (PJB) is a premature discharge that originates in the AV junction either at the level of the AV node or the bundle of His, in the absence of a preceding atrial discharge.

3.3.2 Incidence: PJCs can occur in structurally normal or abnormal hearts and in all age groups. The incidence and prevalence are not well known. Among 16-19 year old males, $0.21 \%$ had premature junctional complexes on 12-lead ECG and similar in other age groups (Hiss and Lamb 1962).

3.3.3 Mechanism: PJCs arise due to increased automaticity or triggered activity due to after depolarizations within the AV junction (Hoffman and Cranefield 1964; Rosen et al. 1980). PJCs may predispose the patient to ventricular tachycardia. Potential causes include normal variants, use of stimulants or alcohol, hypokalemia, hypoxemia, ischemia, and digitalis toxicity (Sherron et al. 1985).

3.3.4 Clinical presentation: It may be incidentally detected or may produce symptoms like palpitations, syncope, or dizziness.

3.3.5 ECG: ECG shows QRS complex morphology similar to sinus node originated activation, without preceding P-wave. A PJC conducting to the ventricle with aberrancy has a QRS complex comparable to PVC. A retrograde P-wave may appear before, during, or after the QRS complex (Sherron et al. 1985) (Figure 4).

3.3.6 Work-up: Depending on the frequency of the PJCs, an ECG can identify the premature complexes. If unsuccessful, a 24-hour Holter monitor may be used. An electrophysiology study maybe needed to identify the location of the origin of the PJCs; origin distal to the AV node can initiate ventricular tachycardia (Sherron et al. 1985).

3.3.7 Management and Prognosis: Patients with PJCs are usually left untreated. The management of underlying causes is essential to decrease symptomatic PJCs. If symptoms are severe, medical management and pacing may be warranted to increase the heart rate.

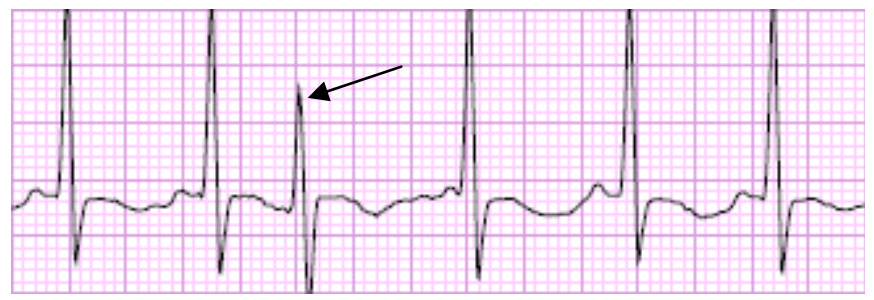

Fig. 4. Premature junctional complex

\section{Bradyarrhythmias}

\subsection{Sinus node dysfunction (SND)}

4.1.1 Definition: The inability of the sino-atrial (SA) node to discharge/activate (sinus pause/arrest) or inappropriately activate (tachycardia-bradycardia syndrome) the atrial tissue. 
4.1.2 Incidence: SND is relatively uncommon in children with normal cardiac anatomy and the exact incidence is unknown. However, it is a common problem in those who have had surgery for congenital heart disease (CHD), especially in patients with Mustard, Senning, and Fontan procedures (Fishberger 2001). Approximately $2 / 3$ of all immediate posttransplant patients have SND (Jacquet et al. 1990).

4.1.3 Mechanism: In sinus node pause/arrest, there is a lack of discharge from the SA node; causing no activation of the atria. With sinus node exit block, the activation may be delayed significantly or blocked completely at the nodal region, without activation of the atria. In tachycardia-bradycardia syndrome, severe sinus bradycardia is followed by tachycardia in the form of atrial flutter in the pediatric population and atrial fibrillation/flutter in adults with repaired congenital heart disease (Duster et al. 1985; Gelatt et al. 1994).

4.1.4 Natural history: Sinus node dysfunction may present as severe sinus bradycardia, sinus pause or arrest, periods of junctional rhythm, and/or alternating tachycardia-bradycardia periods. Sinus node dysfunction increases in frequency with age of the patient and time from surgery.

4.1.5 Clinical presentation: It depends on the age, underlying cardiac conduction abnormality, and hemodynamic status. Although majority of children with structurally normal hearts remain asymptomatic; fatigue, exercise intolerance, palpitations, chest pain, dizziness, and syncope may occur (Fishberger 2001). These symptoms are more frequent in those with corrected congenital heart disease. Infants present with poor feeding, lethargy or heart failure. Sudden death is an uncommon presentation.

4.1.6 ECG: ECG may show severe sinus bradycardia, sinus pause, junctional rhythm, and/or atrial fibrillation/flutter. Due to the short duration, significant episodes can be missed.

4.1.7 Work-up: Non-invasive testing includes a 12-lead ECG, a Holter monitor, an event monitor and exercise stress test. Depending on the frequency of the symptoms and the age group, a 24-hour Holter monitor can be employed. In an older age group with less frequent symptoms, an event recorder can be used to record rhythm abnormalities. In patients who are able to exercise, a treadmill stress test will show lower heart rate response to maximal exercise and rapid decrease in heart rate during recovery time immediately after the test (Martin and Kugler 1999; Fishberger 2001). Intrinsic heart rate (IHR) is the heart rate with complete blockade of autonomic activity with atropine and propranolol (Jose AD and Collison D, 1970). Normal value of IHR is defined as $118.1-(0.57 \times$ age $)$. The intrinsic heart rate decreases with age in complete autonomic blockade but decreases at a faster rate in patients with SND (Benditt et al. 1984). In invasive testing, an electrophysiology study is undertaken to evaluate sinus node automaticity, and conduction time. Sino-atrial conduction time (SACT) and sinus node recovery time (SNRT) are assessed with premature atrial stimulation and atrial overdrive pacing, respectively. The length of SNRT evaluates the sinus node automaticity; prolongation of SNRT indicates suppression of sinus node automatic function.

4.1.8 Management and Prognosis: Medical treatment is dependent on the type of sinus node dysfunction and the presence of associated symptoms. Choice of medications should be selected cautiously and the patient should be monitored closely.

1. Sinus bradycardia and pause/arrest $<3$ sec: no further management; pause/arrest $>3$ sec requires evaluation. In severe sinus bradycardia with hemodynamic instability, sympathomimetic drugs such as atropine and isoproterenol or transcutaneous pacing are used. 
2. Tachycardia-Bradycardia syndrome management is difficult. Drugs used to manage tachycardia may worsen sinus bradycardia and should be used judiciously and may necessitate cardiac pacing.

With failed medical therapy and the need for long-term anti-arrhythmic medications, pacemaker implantation is indicated. According to the 2002 ACC guidelines for pacemaker implantation (Gregaratos 2002), documented symptomatic bradycardia (Class I), symptomatic chronotropic incompetence (Class I), syncope of unknown etiology with electrophysiologic studies suggestive of SND (Class IIa), and chronic awake heart rate $<40$ (Class IIb) are primary indicators for permanent pacing. Permanent pacing is indicated in bradycardia-tachycardia (Class IIa). In refractory or symptomatic SND patients who have failed medical therapy, permanent pacemaker implantation improves clinical status. Pacemaker implantation can provide anti-bradycardia pacing in severe bradycardia; in tachycardia-bradycardia syndrome, pacemaker can provide anti-tachycardia and antibradycardia pacing.

\subsection{First-degree atrio-ventricular heart block}

4.2.1 Definition: Delay in atrio-ventricular conduction at the level of the atrial tissue, AV node, and/or the His-Purkinje system.

4.2.2 Incidence: First degree AV block has been detected in 6\% of neonates (Ferrer 1977). Among 10-13 year old males, 8.5\% had first degree heart block (Scott et al. 1980).

4.2.3 Mechanism: Common mechanism in pediatric patients is hypervagotonia. However, other causes include medications which prolong AV-node refractory period, electrolyte abnormalities, hypothermia, hypothyroidism, rheumatic fever, myocarditis, Lyme disease, congenital heart disease that stretches the atria at the AV node area, cardiac surgery, and myopathies (Weindling 2001). Four sites for first degree AV block with a normal QRS include: atrium (3-20\%) (Sherron et al. 1985), AV node (35-90\%) (Sherron et al. 1985), Bundle of His (15\%) (Sherron et al. 1985) and infra-Hisian conduction system. It is more common in endocardial cushion defects and Ebstein's anomaly of the tricuspid valve due to intraatrial conduction delay (Sherron et al. 1985). Increased vagal tone, calcium-channel blockers, digoxin, and beta-blockers are common causes of AV nodal delay. Quinidine, procainamide, and disopyramide impair phase 0 depolarization and cause delay in bundle of His conduction. Finally, prolongation of conduction in the infra-Hisian region can be caused by sodium-channel blockers as above. With first degree AV block with a wide QRS complex, conduction in the AV node or the bundle of His is delayed; bilateral bundle branch conduction is slowed in most cases; two levels of conduction delay can also be seen (Peuch et al. 1976).

4.2.4 Natural history: First degree heart block has the potential, although rarely, to progress to higher-degree heart blocks in myocarditis, and adults with ischemic heart disease. In a 30year follow up of adult males with moderate P-R interval prolongation, first degree heart block was benign (Mymin et al. 1986).

4.2.5 Clinical presentation: Patients with first degree AV block are asymptomatic unless associated with significant left ventricular (LV) dysfunction. Marked prolongation of the PR interval can cause symptoms mimicking pacemaker-like syndrome.

4.2.6 ECG: There is prolongation of PR interval beyond the normal ranges for age. Normal P$R$ interval naturally varies with age in the pediatric population and prolongation of the P-R interval should be viewed in this context (Figure 5). 
4.2.7 Work-up: An ECG can detect first degree block with or without infranodal disease based on QRS pattern. Atropine and exercise stress testing decrease the vagal tone and enhance AV nodal conduction, thereby shortening the PR interval. If the delay is due to infranodal disease, the conduction delay is worsened. Electrophysiology study can be used to determine the site of conduction delay.

4.2.8 Management and Prognosis: Family history of rhythm or connective tissue disease should be obtained. Repeat ECGs are indicated in those with suspected progression to higher grade blocks. Indications for a pacemaker include first degree block with symptoms suggestive of a pacemaker-like syndrome (Class IIa), and presence of first degree block in neuromuscular diseases and LV dysfunction (Class IIb) (Gregaratos 2002).

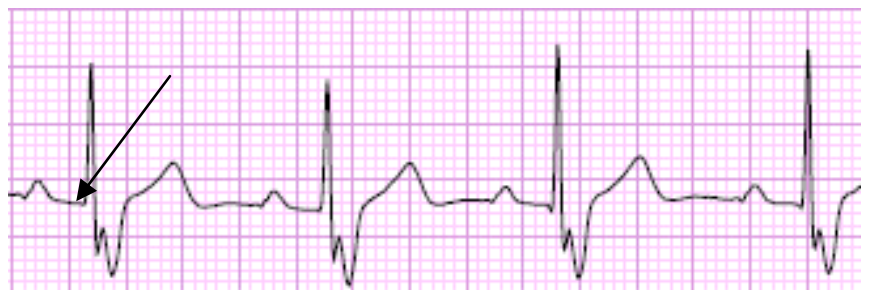

Fig. 5. First degree AV block

\subsection{Second-degree atrio-ventricular heart block}

4.3.1 Definition: Second-degree heart block is atrio-ventricular block in which some atrial discharges are not conducted to the ventricles. It is classified as Mobitz type I (Wenckebach phenomena), Mobitz type II and high grade.

4.3.2 Incidence: Wenckebach phenomena was detected in $2 \%$ of infants, $7 \%$ of toddlers and $6 \%$ of children at rest (von Bernuth et al. 1989).

4.3.3 Mechanism: Mobitz type I (Wenckebach) block occurs at the AV node and His-Purkinje system in a ratio of 3:1, respectively (Peuch et al. 1976). Wenckebach phenomenon results from hypervagotonia in athletes and young subjects (Stein et al. 2002). Previous cardiac surgery and ischemic heart disease in adulthood are associated with Wenckebach phenomena. Mobitz type II block occurs infra-nodally in most cases (Dhingra et al. 1974).

4.3.4 Natural history: Mobitz type I block is usually benign. However, in pediatric population without heart disease, Mobitz type I block may serve as intermediary prior to development of idiopathic complete heart block (Young et al. 1977). In adults, second degree AV-nodal block proximal to the His bundle has a benign course in those without heart disease; but is associated with poor prognosis in those with underlying heart disease (Strasberg et al. 1981). Mobitz type II can progress to symptomatic complete heart block and is associated with Stokes-Adams attacks and sudden death (Dhingra et al. 1974).

4.3.5 Clinical presentation: Mobitz type I block is usually well tolerated; but may present with significant bradycardia. Patients with Mobitz type II block may be asymptomatic; but can present with bradycardia, exercise intolerance, syncope, postural hypotension, and sudden death.

4.3.6 ECG: Mobitz type I shows progressive prolongation of PR interval followed by a nonconducted atrial discharge (Figure 6a). Mobitz type II is characterized by stable PR interval with intermittent failed conduction (Figure 6b). PR interval can be normal or prolonged. 
4.3.7 Work-up: ECG is diagnostic. If ECG is inconclusive, a 24-hour monitor is suggested. If there is one conducted beat in a cycle as in a 2:1 block, it is difficult to differentiate between Mobitz types I and II blocks; atropine can be used to elicit a 3:2 conduction in Mobitz type I block. Response to atropine suggests the block to be at or proximal to the AV node. If exercise initiates or exacerbates Mobitz type I block, infranodal location of the block is suspected. An electrophysiology study is indicated if there is unexplained syncope, and AV block distal to the AV node, to identify the location of the block (Zipes et al. 1995). In complete or high grade second degree AV block, an echocardiogram is indicated (Cheitlin et al. 2003).

4.3.8 Management: Medical management of Mobitz type I includes identification and treatment of underlying causes. Sympathomimetic agents such as scopolamine, theophylline, or glycopyrrolate can be used in symptomatic bradycardia secondary to increased vagal tone. In patients with Mobitz type II, syncope is a poor prognostic factor (Dhingra et al. 1974). Symptomatic chronic bradycardia in Mobitz type I and type II (Class I), Mobitz type II with bifascicular or trifascicular block (Class I), Mobitz type II with wide QRS (Class I), asymptomatic Mobitz type II (Class IIa), and neuromuscular disease with AV block (Class IIb) are all indications for permanent pacemaker implantation (Gregaratos 2002).

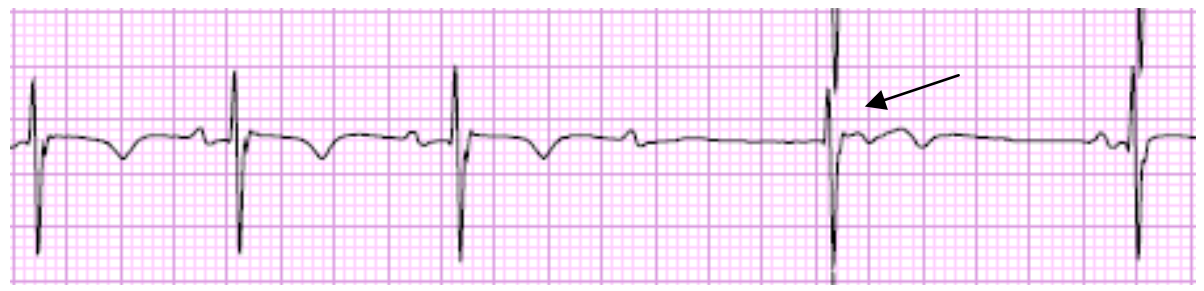

Fig. 6.a Second degree Type I AV block (Wenckebach)

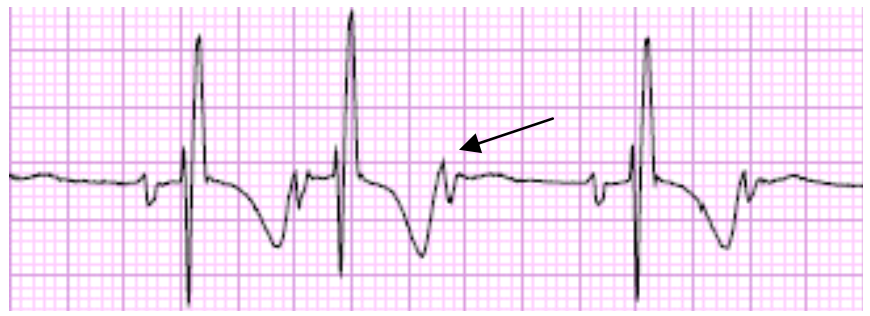

Fig. 6.b Second degree Type II (Mobitz) AV block

\subsection{Third-degree (Complete) Atrio-Ventricular Heart Block}

4.4.1 Definition: Complete failure of conduction of atrial impulses to the ventricles.

4.4.2 Incidence: Complete heart block may be acquired or congenital. The most common cause of acquired complete heart block in pediatric patients is surgery for left ventricular outflow tract obstruction, repair of corrected transposition of the great arteries (L-TGA), ventricular septal defects and TOF. Acquired complete AV block is also seen in Lyme carditis, myocarditis, myopathies, and metabolic diseases. Congenital complete heart block occurs 1 per 15,000 to 1 per 22,000 live births and is often associated with maternal 
connective tissue disease, in particular systemic lupus erythematosis (Michaelsson and Engle 1972). Spontaneous complete heart block is seen in left atrial isomerism, endocardial cushion defects and in $20 \%$ of patients with L-TGA (Huhta et al. 1983; Lundstrom et al. 1990).

4.4.3 Mechanism: Possible mechanisms include abnormal development of the conduction system and the AV node, or acquired most commonly after cardiac surgery. The escape rhythms below the AV node take over when the atrial discharge is not conducted to the ventricles. The rate of the escape rhythm correlates with the level of the block, with the proximal sites having a higher rate.

4.4.4 Natural history: Complete heart block is associated with L-TGA and septal defects, as well as sequelae of maternal connective tissue. Mortality is highest during the neonatal period (Roberts and Gillette 1977). Congenital complete heart block in structurally normal heart, when uncorrected, leads to cardiac enlargement with impaired ventricular systolic function (Beaufort-Krol et al. 2007). There is a significant correlation between persistent heart rate of 50 or less and incidence of syncope, pre-syncope, and/or sudden death (Karpawich et al. 1981; Dewey et al. 1987).

4.4.5 Clinical presentation: Many patients are asymptomatic in early life. Symptoms range from none to palpitations, chest pain, weakness, syncope, exercise intolerance, dizziness, and congestive heart failure in congenital complete heart block. Symptoms are usually dependent on ventricular rate, frequency of premature ventricular beats, and atrioventricular synchrony.

4.4.6 ECG: Complete heart block is depicted by atrio-ventricular conduction blockade. In patients with normal hearts, the QRS complex is narrow if the block is at the AV node or His bundle; the QRS complex is wide if the block occurs after the bifurcation of the bundle of His (Weindling 2001). An ECG should be used to differentiate complete heart block from accelerated junctional rhythm or severe sinus bradycardia with appropriate junctional escape rate (Weindling 2001). Complete heart block is associated with a prior lesser-degree block (25\%) and accompanied or preceded by bundle branch block (Levine et al. 1956). Associated prolongation of QT interval is seen in about $18 \%$ of congenital complete heart block (Figure 7).

4.4.7 Work-up: A 12-lead ECG establishes the diagnosis. In symptomatic patients treated with a pacemaker and with a suspicion of another arrhythmia (Class I), and patients with premature, concealed junctional depolarizations as in pseudo-AV block (Class II), an electrophysiology study is indicated (Zipes et al. 1995). In patients with complete AV block or advanced second-degree AV block, an echocardiogram is used to visualize cardiac function, size, structure, and valvular regurgitation (Cheitlin et al. 2003; Beaufort-Krol et al. 2007). Exercise stress testing in asymptomatic patients with congenital complete heart block is used to determine degree of exercise tolerance and arrhythmias associated with exercise (Gibbons et al. 2002).

4.4.8 Management and Prognosis: Primary mode of management of patients with complete heart block is pacemaker implantation. The time of pacemaker implantation is controversial. Third degree AV block with symptomatic bradycardia, post-operative complete heart block that does not resolve in 7-10 days, and congenital complete heart block with wide QRS escape rhythm and ventricular dysfunction, and congenital complete heart block with rate $<50 \mathrm{bpm}$ are Class I indications for pacemaker implantation (Gregaratos 2002). Prognosis for neonates and infants post pacemaker implantation is very good (Aellig et al. 2007). 


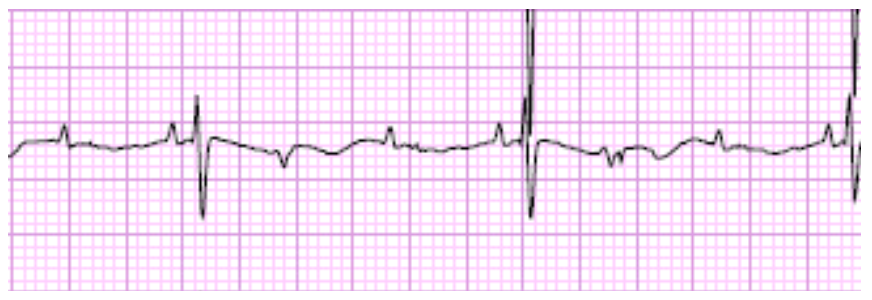

Fig. 7. Third degree (complete) AV block

\subsection{Atrial escape rhythm}

4.5.1 Definition: Atrial escape rhythm arises from ectopic atrial foci during periods of severe sinus bradycardia or significant sinus node dysfunction. These atrial ectopic foci have slower spontaneous rates and are unstable. The exact incidence of atrial escape rhythm is not known

4.5.2 Mechanism: The automaticity of an ectopic atrial focus generates a faster rate than the dysfunctional sinus node by escaping overdrive suppression of the SA node. Atrial escape rhythm rate is between 60-80 beats per minute (Dubin 2000).

4.5.3 Natural history: Atrial escape rhythm occurs in sinus node dysfunction and severe sinus bradycardia. Resolution or treatment of a slow underlying sinus rhythm resolves the atrial escape rhythm. Automaticity in ectopic foci are more responsive to autonomic control compared to the SA node (Randall et al. 1981).

4.5.4 Clinical presentation: Most symptoms are secondary to underlying rhythm abnormalities such as sinus node dysfunction with severe sinus bradycardia, sinus pause and/or sinus arrest. These include dizziness, exercise intolerance, and possible syncope.

4.5.5 ECG: The P-wave morphology differs from the sinus P-wave. The P-wave is smaller and shorter in duration with the P-wave axis dependent on the location of the ectopic focus. The PR interval may be shorter than the normal PR interval due to the proximity of the ectopic atrial focus to the AV node. The atrial escape rhythm is slower than the normal sinus rate.

4.5.6 Work-up: Work-up is focused on identifying the underlying abnormality leading to increased automaticity or suppression of the sinus node.

4.5.7 Management and Prognosis: In symptomatic patients, slow sinus rhythm is managed with sympathomimetic agents. If unresponsive to medical treatment, pacemaker implantation may be necessary for management of sinus node dysfunction or severe sinus bradycardia.

\subsection{Atrio-ventricular junctional escape rhythm}

4.6.1 Definition: AV junctional escape rhythm is defined as an ectopic rhythm starting in the $\mathrm{AV}$ junction due to failure of the atrial impulse to conduct. The ectopic rhythm may originate between the AV node and the bundle of His.

4.6.2 Incidence: Episodes of junctional escape rhythm were reported in 19\% of full-term neonates (Southall et al; Southall et al. 1980).

4.6.3 Mechanism: AV junctional escape rhythm occurs due to enhanced automaticity or a reentry mechanism. The ectopic focus can occur below the AV node and at or above the bundle of His, in the absence or suppression of an SA node or atrial impulse (Gamble et al. 2007). Due to the lack of overdrive suppression from the higher pacemakers, the AV 
junction has its intrinsic automaticity. The junctional escape rate ranges between $40-60$ beats per minute (Dubin 2000).

4.6.4 Natural history: The junctional pacemaker can exist in the region of the distal AV node or the proximal portion of the His-bundle near the AV-node (Alison et al. 1995). Nodal escape rhythm is associated with apnea (Valimaki and Tarlo 1971). The rate of AV junctional escape rhythm varies with underlying conditions, such as sick sinus syndrome, complete heart block, ablation of AV node, congenital heart surgery, hypertrophic cardiomyopathy, and ischemia (Kleinfeld and Boal 1978; Alison et al. 1995; Shepard et al. 1998). Inflammation, hypoxia, metabolic, drugs, or electrolyte disturbances are implicated in the etiology of junctional rhythms (Fisch and Knoebel 1970).

4.6.5 Clinical presentation: Symptoms pertaining to AV junctional escape rhythm are related to suppression of the sino-atrial tissues and or failure to conduct to the junction. Symptoms include dizziness, exercise intolerance, near syncope or syncope.

4.6.6 ECG: In AV junctional escape rhythm, there are no P-waves before the QRS complexes; they may occur simultaneously or follow the QRS complexes. The QRS complex is narrow if the focus is closer to the AV node or wide if proximal to the His bundle bifurcation. In the case of complete AV block, P-waves of sinus origin are dissociated from the QRS complexes. 4.6.7 Work-up: ECG may identify the rhythm; if unsuccessful, a Holter monitor can help identify the rhythm abnormality. Workup should also include identifying the causes listed above.

4.6.8 Management and Prognosis: Pacemaker implantation may be needed in symptomatic bradycardia due to junctional escape rhythm.

\subsection{Idioventricular escape rhythm}

4.7.1 Definition: Ventricular escape (idioventricular) rhythm arises below the bifurcation of the His bundle. The ventricular escape results when the higher pacemaker systems are unable to generate and/or conduct an impulse to the ventricles. It is rare and incidence is not known.

4.7.2 Mechanism: The automaticity of the ventricular myocardium is pronounced in the absence of supraventricular impulses. The overdrive suppression from higher pacemaker centers is absent, leading to the automaticity of the ventricular focus to conduct to the ventricles. Idioventricular rate ranges from 20-40 beats per minute (Dubin 2000).

4.7.3 Natural history: Idioventricular rhythm is seen with hypothermia, drugs, and ischemia (Talbot and Greaves 1976).

4.7.4 Clinical presentation: Stokes-Adams syncopal attacks are more prevalent in ventricular escape rhythm than atrial or junctional escape rhythms. This is also accompanied by dizziness and exercise intolerance.

4.7.5 ECG: The ECG shows wide QRS complexes with a rate of 20-40 beats per minute. If Pwaves exist, they occur independently and are dissociated from the ventricular beats.

4.7.6 Work-up: ECG is the first step in diagnosing ventricular escape rhythm. Holter and event recorders are alternatives when ECG is not diagnostic. Electrophysiology study is indicated (class IIA) in patients with suspected bradyarrhythmias with syncope (Zipes 2006). An EP study can establish the level of the AV block.

4.7.7 Management and Prognosis: Symptomatic relief is important in patients with ventricular escape rhythm. Pacemaker implantation with ventricular pacing is essential to avoid StokesAdams attacks. 


\section{Tachy-arrhythmias}

\subsection{Atrial tachy-arrhythmias \\ 5.1.1 Physiologic (appropriate) sinus tachycardia}

5.1.1.1 Definition: Sinus tachycardia is an automatic rhythm characterized by rapid discharge from the sinus node due to physiologic influences (Walsh 2001b; Blomstrom-Lundqvist et al. 2003; Blomstrom-Lundqvist 2003). The rate of sinus tachycardia is dependent on age.

5.1.1.2 Incidence: Dependent on the body's increased sympathetic response to physiologic phenomena, such as fever, dehydration, anxiety, pain, medications, anemia, hyperthyroidism and heart failure.

5.1.1.3 Mechanism: Occurs due to increased automaticity with rate variability associated with increased sympathetic response or vagal withdrawal.

5.1.1.4 Natural history: Decreasing the underlying enhanced sympathetic tone or increasing the vagal tone will decrease the sinus tachycardia.

5.1.1.5 Clinical presentation: The inciting factor for sympathetic response or vagal withdrawal is the usual presenting symptom. Very high rate of sinus tachycardia can compromise cardiac output by decreasing the ventricular filling time.

5.1.1.6 ECG: There is a normal P-wave axis and a P-wave precedes each QRS complex (Van Hare 1999). P-waves may have larger amplitude and become peaked (Blomstrom-Lundqvist 2003).

5.1.1.7 Work-up: Identify the extrinsic factors causing the tachycardia and treat them. It is also necessary to rule out inappropriate sinus tachycardia (see below).

5.1.1.8 Management and Prognosis: Prognosis depends on the nature of the underlying cause. No specific treatment is required for physiologic sinus tachycardia except treatment of the underlying causes. Beta-adrenergic receptor blockers may occasionally be used for treatment of physiologic symptomatic sinus tachycardia (Blomstrom-Lundqvist 2003).

\subsubsection{Inappropriate Sinus Tachycardia (IST)}

5.1.2.1 Definition: Elevated resting heart rate for the physiological state and/or an exaggerated heart rate response to exercise or stress (Krahn et al. 1995; Blomstrom-Lundqvist 2003).

5.1.2.2 Incidence: IST often follows a viral illness or physical trauma, usually in women (90\%) who are generally healthy physically and emotionally (Fogoros 2006).

5.1.2.3 Mechanism: Etiology is not known. The mechanism may involve a primary sino-atrial (SA) node disorder with increased automaticity, a dysautonomia with increased sympathetic tone, decreased parasympathetic tone, and/or increased SA node betaadrenergic sensitivity, or impaired baroreflex control (Krahn et al. 1995; Fogoros 2006; Morillo and Guzman 2007). Another mechanism is stimulation of the beta-adrenergic receptors by anti-beta adrenergic autoantibodies (Chiale et al. 2006).

5.1.2.4 Natural history: IST is more common in women in the 20 s and 30 s, who are otherwise healthy. It is likely to improve over time (Fogoros 2006).

5.1.2.5 Clinical presentation: Palpitations, lightheadedness, exercise intolerance, and fatigue are common symptoms. Patients may also have orthostatic intolerance, chest pain, anxiety, depression, headache and myalgia, gastro-intestinal disturbances, and diaphoresis (Shen 2005; Fogoros 2006). Only consistent finding on physical exam is tachycardia (Shen 2005; Fogoros 2006).

5.1.2.6 ECG: P-wave morphology and axis is similar to that of normal sinus rhythm (Shen 2005). There is tachycardia at rest on ECG. 
5.1.2.7 Diagnosis: Criteria for diagnosis include: a) persistent sinus tachycardia during the day with increased rate in response to activity and normalization during sleep per 24-hour Holter recording, b) non-paroxysmal tachycardia and symptoms, c) P-wave morphology and activation similar to sinus rhythm, and d) exclusion of secondary causes of sinus tachycardia (Blomstrom-Lundqvist 2003).

5.1.2.8 Work-up: In addition to the ECG, a 24-holter monitor will document elevated mean heart rates above normal for age, elevated daytime heart rates, and/or exaggerated elevation in sinus rate from supine to upright position (Shen 2005). A diagnostic electrophysiology (EP) study should be considered if the etiology of the tachycardia is unclear (to rule out sinus node re-entry tachycardia). EP study consistent with IST would include slow warm up and cool down phases suggesting sinus node involvement, surface Pwave similar to that of normal sinus rhythm, and earliest activation arising near the sinus node area along the crista terminalis during mapping. IST is not initiated with programmed stimulation of the atria (Lin and Callans 2004). In addition to electrophysiology testing, there is also a need for autonomic dysregulation testing. Neurologic, cardiovascular rehabilitation, and psychiatric consultations are often necessary because IST is a diagnosis of exclusion (Shen 2005).

5.1.2.9 Management and Prognosis: Treatment involves a multidisciplinary approach. Medications including beta blockers, calcium channel blockers and class IC anti-arrhythmic agents decrease SA node automaticity (Chiale et al. 2006). However in patients with IST secondary to autonomic dysregulation, control of heart rate does not always lead to resolution of symptoms. In patients with no evidence of autonomic dysregulation if biofeedback, meditation, and medical therapy fail, superolateral crista terminalis ablation could be considered (Shen 2005; Fogoros 2006). Risks of RF ablation include SVC syndrome, diaphragmatic paralysis and persistent junctional rhythm. The prognosis of IST is benign over a mean follow-up of 6 years (Still et al. 2005).

\subsubsection{Sinus Node Re-entry Tachycardia}

5.1.3.1 Definition: Sino-atrial node re-entry tachycardia (SNRT) is a paroxysmal arrhythmia characterized by a re-entry mechanism that is located entirely within the SA node or the perisinus atrial tissue (Lin and Callans 2004).

5.1.3.2 Incidence: Incidence of SNRT is underestimated due to lack of symptoms very often. In patients undergoing electrophysiology study for supraventricular tachycardia, incidence ranges from 1.8\%-16.9\% (Blomstrom-Lundqvist 2003). Incidence of SNRT is higher $(10 \%)$ in those with underlying organic heart disease (Garson Jr. and Gillette 1981).

5.1.3.3 Mechanism: A re-entry mechanism is present but it is not known whether the entire circuit is within the SA node or involves perisinus atrial tissue.

5.1.3.4 Clinical presentation: Patients are often asymptomatic. Symptoms include palpitations, lightheadedness, presyncope, and rarely syncope (Gomes et al. 1985).

5.1.3.5 ECG: SNRT heart rate ranges from $80-200$ beats/min. P-wave axis and morphology are similar to that of normal sinus rhythm. P-R interval is shorter than R-P interval. An atrio-ventricular (AV) nodal Wenckebach block may be present (Olgin and Zipes 2005a).

5.1.3.6 Diagnosis: Criteria for diagnosis include: a) paroxysmal tachycardia, b) P-wave morphology and endocardial atrial activation similar to that of normal sinus rhythm, c) inducibility with programmed premature atrial stimuli irrespective of the location of stimulation or the AV junction, d) the ability to terminate the arrhythmia with atrial 
premature beat or atrial pacing, and e) termination with vagal maneuvers or adenosine (Narula 1974; Blomstrom-Lundqvist 2003).

5.1.3.7 Work-up: Electrophysiology study is indicated in patients with frequent and poorlytolerated episodes of tachycardia, unclear mechanism of tachycardia, and refractoriness to medications.

5.1.3.8 Management and Prognosis: Vagal maneuvers or adenosine may interrupt the tachycardia. Acutely, atrial pacing is effective. Beta blockers, calcium channel blockers, amiodarone, and digitalis can benefit patients with increased frequency of symptoms. However, prophylactic treatment of SNRT is not recommended. In patients undergoing an electrophysiology study, catheter ablation can be successful (Goya et al. 1999).

\subsubsection{Ectopic (focal) Atrial Tachycardia}

5.1.4.1 Definition: Ectopic or focal atrial tachycardia (EAT) is a tachycardia mediated by inappropriate atrial impulse generation from a single atrial focus outside of the SA node (Walsh 2001a).

5.1.4.2 Incidence: EAT is commonly found in children and teenagers and makes up approximately $15 \%$ of all newly diagnosed supraventricular tachycardias across all ages (Walsh 2001a). Both genders are equally affected. Prevalence of EAT is $0.34 \%$ in asymptomatic male patients and $0.46 \%$ in symptomatic male patients (Poutiainen et al. 1999).

5.1.4.3 Mechanism: EAT can be due to three mechanisms: a) enhanced automaticity, b) triggered activity, and c) microreentry (Roberts-Thomson et al. 2006). The tachycardia cannot be initiated or terminated with programmed stimuli (Walsh 2001a). However, the exact mechanism is difficult to ascertain.

5.1.4.4 Natural history: In the majority of patients, the course is benign. Persistent incessant EAT can lead to ventricular dysfunction, congestive heart failure and low cardiac output states, termed tachycardia-mediated cardiomyopathy. However, termination of the rhythm can lead to improvement in ventricular function (Packer et al. 1986; Naheed et al. 1995). There is slowing of heart rate with time, with reversion to sinus rhythm or change in P-wave morphology (Poutiainen et al. 1999).

5.1.4.5 Clinical presentation: Young patients are often asymptomatic until onset of left ventricular dysfunction. Patients may also have palpitations, lightheadedness, and exercise intolerance even with normal LV function (Walsh 2001a).

5.1.4.6 ECG: ECG shows a supraventricular tachycardia with rates within normal range to 300 beats per minute. ECG criteria for diagnosis include: a) the P-wave morphology and axis different than that of normal sinus rhythm, b) acceleration after initiation and deceleration before termination, c) unaffected by presence of an atrio-ventricular block, and d) an initial P-wave of the tachycardia similar to the subsequent P-waves (Olgin and Zipes 2005a). The QRS complex is similar to that of sinus rhythm.

5.1.4.7 Work-up: The location of the ectopic focus can be determined by noting P-wave frontal plane axis. "Warm-up" and "cool-down" patterns can be appreciated and suggestive of an automatic mechanism. AV-block (Mobitz 1) can be appreciated episodically during sleep. Adenosine administration will demonstrate AV-block with continuous marching of Pwaves (Figure 8). When the ectopic focus is close to the sinus node and/or there is poor ventricular function, electrophysiology testing is necessary to differentiate between sinus tachycardia, atrial flutter, and ectopic atrial tachycardia. Difficulty to terminate tachycardia 
with pacing maneuvers and external cardioversion are suggestive of EAT (Walsh 2001a). Echocardiography at the time of diagnosis is important to document the ventricular function (Naheed et al. 1995).

5.1.4.8 Management and Prognosis: Paroxysmal and incessant atrial tachycardia are difficult to treat medically (Blomstrom-Lundqvist 2003). Acute therapy includes IV verapamil and IV beta blockers. There is no termination of tachycardia with atrial pacing, except for mild slowing of the heart rate. DC cardioversion is usually not effective but may be effective in those with micro-re-entry mechanism. Class IA (e.g. quinidine) and IC (e.g. flecainide, propafenone) antiarrhythmics are indicated for those without cardiac failure and Class III (amiodarone) is recommended in those with poor ventricular function (Blomstrom-Lundqvist 2003). Chronic therapy includes calcium channel blockers, Classes IA, IC, and III anti-arrhythmics; Class IC is not indicated in those with coronary artery disease. Intravenous amiodarone or oral sotalol can reduce rates and restore sinus rhythm (Skinner and Sharland 2008). Catheter ablation is successful in $86-90 \%$ with recurrence rate of $8 \%$ (Walsh 2001a; Hsieh and Chen 2002). In children, $75 \%$ had restoration of normal sinus rhythm after medical therapy (Naheed et al. 1995). In patients with drug refractory EAT or incessant AT with tachycardia-induced cardiomyopathy, best option is ablation of the focus (Blomstrom-Lundqvist 2003). Because digitalis toxicity can elicit atrial tachycardia, check digitalis levels and avoid hypokalemia.
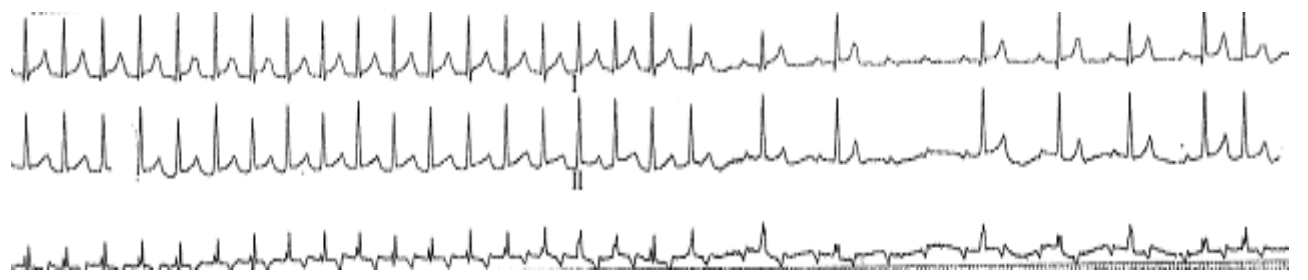

Fig. 8. Ectopic atrial tachycardia with effect of adenosine

\subsubsection{Multifocal (chaotic) Atrial Tachycardia}

5.1.5.1 Definition: Tachycardia characterized by electrocardiographic finding of multiple (at least three) distinct P-wave morphologies with irregular P-P intervals, isoelectric baseline between $\mathrm{P}$-waves and ventricular rate $>100$ beats/min (Blomstrom-Lundqvist 2003).

5.1.5.2 Incidence: MAT is a rare entity. In the pediatric age group, it is reported exclusively in neonates and infants, mostly associated with a normal heart (Kastor 1990). It is also associated with post-operative congenital heart disease, hypertrophic and dilated cardiomyopathies, and unrepaired atrial septal defects (Walsh 2001a). Incidence is $0.2 \%$ among infants presenting with new onset arrhythmias (Salim et al. 1995). It is associated with significant respiratory disease in adults. It is also associated with hypoxemia, glucose intolerance, hypokalemia, digitalis, and chronic renal failure (Kones et al. 1974).

5.1.5.3 Mechanism: Exact mechanism of MAT is unclear; however, automaticity versus triggered activity is postulated (Walsh 2001a).

5.1.5.4 Natural history: Spontaneous resolution may occur in $50-80 \%$ of patients with MAT by 12-18 months of age (Sokoloski 1999). There is no recurrence of MAT in infants with normal cardiac structure (Walsh 2001a).

5.1.5.6 Clinical presentation: Most patients are asymptomatic at the time of detection. If tachycardia is persistent, cardiomyopathy develops and symptoms consistent with cardiomyopathy arise. 
5.1.5.7 ECG: Diagnostic criteria include: 1) irregular atrial rates, 2) at least three different Pwave configurations, 3) isoelectric baseline between discrete P waves, 4) irregular P-P, P-R, and R-R intervals, and 5) absence of a dominant atrial pacemaker (Sokoloski 1999). Atrial rate may range from $150-500$ beats/minute.

5.1.5.8 Work-up: Meeting ECG criteria is sufficient for diagnosis of MAT (figure 9).

5.1.5.9 Management and Prognosis: It is usually not responsive to DC cardioversion, vagal maneuvers, or IV antiarrhythmics. However, digoxin, beta blockers, amiodarone, and flecainide are options to consider. Calcium channel blockers are not indicated in neonates and infants less than one year of age. When drug therapy is successful, it should be continued for 6-12 months. In rare cases with medication refractory MAT, multiple foci ablation is required. In refractory cases, AV node ablation with ventricular pacemaker implantation may be necessary (Walsh 2001a).

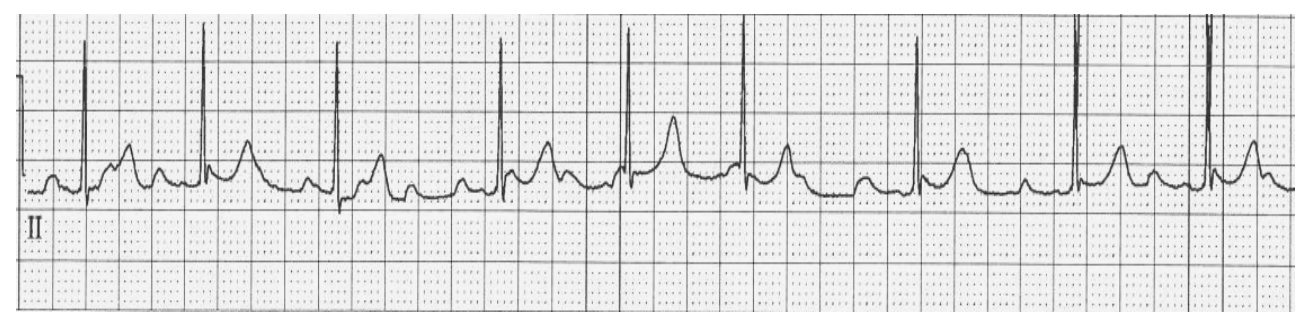

Fig. 9. Multifocal atrial tachycardia

\subsubsection{Atrial flutter}

5.1.6.1 Definition: Atrial flutter is a regular atrial tachycardia due to a macro-reentrant rhythm confined to the atrium. There are two types of atrial flutter: 1) typical or Type I and 2) atypical or Type II. Typical atrial flutter is the most common, cavo-tricuspid isthmusdependent, terminated by rapid atrial pacing and is further divided into counter-clockwise and clockwise circuits. Atypical atrial flutter has a faster rate ( $>350$ beats/minute), and is not cavo-tricuspid isthmus-dependent (Saoudi et al. 2001; Waldo 2004). Left atrial flutter is also considered to be atypical (Garan 2008).

5.1.6.2 Incidence: In a study of 380 patients with first flutter episode between 1 and 25 years of life, $81 \%$ had congenital heart disease, and $8 \%$ had normal hearts (Garson Jr. et al. 1985). Among adults, the incidence of atrial flutter overall was 88 per 100,000 person-years with male predominance (4:1) and is noted in patients with heart failure, pulmonary disease, thyrotoxicosis, post repair of congenital heart defects, and mitral valve disease (Granada et al. 2000; Waldo 2004). The incidence of atypical atrial flutter is rare in patients without prior cardiac surgery. Atypical right atrial flutter occurs in $8 \%$ of patients with atrial flutter (Yang et al. 2001). Persistent or recurrent atrial flutter is common in patients post-cardiac surgery (Waldo 2004).

5.1.6.3 Mechanism: The mechanism of atrial flutter is re-entry with an excitable gap, within the atrium, right more than left (Waldo 2004). The flutter circuit requires an initially activated region to repolarize as the action potential travels through the slow conduction tissue and reactivates the initial part, thereby creating a re-entry circuit. The atrial activation along the circuit proceeds from the coronary sinus superiorly to the high right atrium area and craniocaudally along the free wall and medially to the isthmus between the tricuspid valve and the inferior vena cava in a counter-clockwise manner. There is an area of slow 
conduction in the posteroinferior aspect of the circuit. The clockwise or reverse typical circuit proceeds in the opposite direction (Sokoloski 1999).

5.1.6.4 Natural history: In patients less than one year of age and with no previous cardiac surgery, atrial flutter spontaneously converts in $26 \%$. Congestive heart failure was more prevalent in those with atrial flutter of long duration. Of the infants with decreased ventricular function, all recovered normal function after conversion to sinus rhythm. If patients had additional arrhythmia, recurrence of atrial flutter was more likely (Texter et al. 2006). Atrial flutter persisting over a long period of time can evolve into atrial fibrillation (Waldo 2004).

5.1.6.5 Clinical presentation: In neonates, $80 \%$ are asymptomatic and $20 \%$ may present with congestive heart failure (Texter et al. 2006). Older patients can experience palpitations, dizziness, chest tightness, chest pain, shortness of breath, and fatigue. Hypotension, poor exercise tolerance, congestive heart failure and impaired cardiac output can also result from atrial flutter (Blomstrom-Lundqvist 2003; Andrew and Montenero 2007).

5.1.6.6 ECG: Counterclockwise (typical) atrial flutter has negative flutter $(\mathrm{F})$ waves in the inferior leads II, III, and aVF and positive F waves in Lead V1 (figure 10). Clockwise (reverse typical) atrial flutter shows positive $\mathrm{F}$ waves in the inferior leads II, III, and aVF and negative F waves in lead V1 (Blomstrom-Lundqvist 2003). In typical atrial flutter, P waves are absent. The flutter rate can range from $240-340$ beats/minute with no isoelectric interval between consecutive waves. Flutter rates in infants can be as high as 580 beats/minute with rapid ventricular response rates of 200 beats/minute with 2:1 AV conduction in $75 \%$ and variable block in the remainder (Texter et al. 2006). Flutter waves typically have 90-degree axis. The QRS complexes appear normal unless there is rate-dependent aberrancy or block.

5.1.6.7 Work-up: Echocardiogram is indicated in those with congestive heart failure to evaluate ventricular function and structure.

5.1.6.8 Management and Prognosis: Management of atrial flutter is accomplished through electrical cardioversion, rapid atrial pacing, pharmacological therapy, and catheter ablation. The goal of flutter management is to decrease the ventricular rate and to restore normal sinus rhythm. Chronic anti-arrhythmic therapy may not be needed in those with uncomplicated, asymptomatic atrial flutter after conversion to sinus rhythm (Texter et al. 2006; Skinner and Sharland 2008). Drugs are successful in controlling atrial flutter in about $58 \%$ of young patients. Amiodarone, digoxin, and propranolol are found to be effective (Garson Jr. et al. 1985). Atrial flutter causing hemodynamic instability should be terminated acutely with electrical cardioversion (2J/Kg) (Class 1 level of evidence) (BlomstromLundqvist 2003; Texter et al. 2006). Although cardioversion is successful acutely, atrial flutter recurs frequently. Recurrences of atrial flutter can be prevented by administering Class IA, IC, and III anti-arrhythmic agents. Ventricular rate can be controlled with AVnodal blockers such as digitalis and class II (beta blockers), III (amiodarone, sotalol), and IV (calcium channel blockers) anti-arrhythmic agents. Flecainide (Class IC anti-arrhythmic) should be administered with an AV-node blocking agent. As flecainide slows the atrial rate, it may facilitate 1:1 AV conduction, leading to clinical compromise (Skinner and Sharland 2008). Calcium channel antagonists are contraindicated in children less than one year of age. When atrial flutter is refractory to medical management, transesophageal atrial overdrive pacing (TEAP) and catheter ablation are excellent options. In a study by Ajisaka (1997), paroxysmal atrial flutter was terminated in greater than 50 percent of the adult subjects with low-output, short-duration TEAP (Ajisaka et al. 1997). In young adults with repaired congenital heart disease, $50-88 \%$ had successful ablation of recurrent atrial flutter 
(Blomstrom-Lundqvist 2003). Catheter ablation is used to create a bidirectional conduction block across the cavo-tricuspid isthmus. In addition to palliating with catheter ablation, underlying causes of atrial flutter such as stimulants and sympathomimetic agents should also be eliminated.

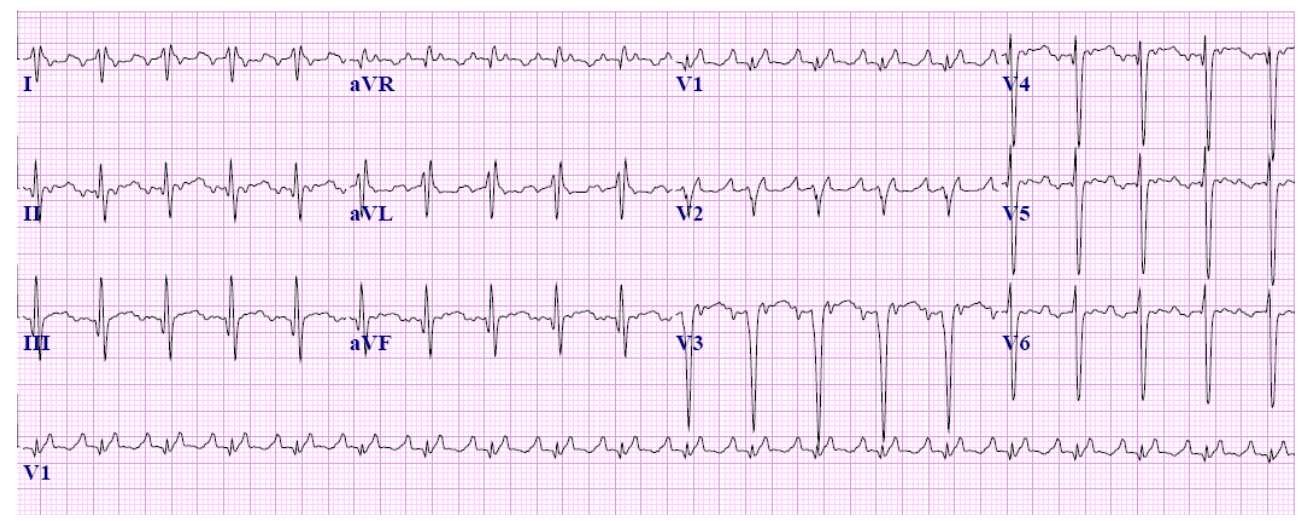

Fig. 10. Atrial flutter

\subsubsection{Intra-atrial Reentry Tachycardia}

5.1.7.1 Definition: Intra-atrial reentry tachycardia (IART) is a group of reentry tachycardia associated with repaired or unrepaired congenital heart disease (CHD). It is also known as slow atrial flutter, incisional atrial tachycardia, and macro-reentrant atrial tachycardia. It is very often a long-term complication of cardiac surgery involving the atrium (Triedman 2001). It is frequently associated with Fontan, Mustard, Senning, repaired tetralogy of Fallot, and TAPVR repairs. Risk factors for IART are older age of surgery and long-term follow-up. 5.1.7.2 Incidence: Incidence of IART often depends on the congenital heart disease and the surgery preceding the onset. Structural heart disease is evident in about $89 \%$ of patients with IART (Haines and DiMarco 1990).

5.1.7.3 Mechanism: IART has a re-entrant mechanism defined within the atrium and does not necessarily follow the atrial flutter circuit. Majority of the reentrant circuits are mainly confined to the right atrium (Triedman 2001).

5.1.7.4 Natural history: IART is intimately associated with atrial fibrillation and thromboembolic phenomena. Natural history is associated with increased frequency of recurrences, regardless of treatment with anti-arrhythmic medications or ablation (Triedman 2004). Intermediate follow-up post ablation of IART shows frequent recurrence with new IART configurations (Triedman et al. 1997).

5.1.7.5 Clinical presentation: Symptoms in patients with IART can range from being asymptomatic to congestive heart failure to significant cardiovascular compromise, including sudden cardiac death (Triedman 2004). Sinus node dysfunction with symptomatic bradycardia is a common association with IART.

5.1.7.6 ECG: Flutter morphology or uniform $P$ waves are frequently noted with constant cycle length longer than typical atrial flutter (figure 11). Multiple ECG morphologies can be obtained from a single patient due to various activation mechanisms. There is often 1:1 AV conduction based on the cycle length. There is sudden onset and termination with pacing and is entrainable (Triedman 2001; Triedman 2004). 
5.1.7.7 Work-up: In refractory atrial reentry arrhythmias, an electrophysiology study must be conducted to further elucidate the nature of the substrate responsible for IART.

5.1.7.8 Management and Prognosis: Acute management in cardiovascular compromise consists of direct current (DC) cardioversion. Other options in clinically stable patients include antiarrhythmic medications, atrial overdrive pacing, and anti-tachycardia pacing with pacemakers and transesophageal pacing. For chronic therapy, sotalol and amiodarone have been reported to be effective in approximately $60 \%$ (Triedman 2001). Implanted anti-tachycardia pacemakers provide relief from symptomatic tachycardia and bradycardia associated with sinus node dysfunction. In cases refractory to medical management, catheter ablation can be attempted. Intra-operative surgical Maze procedure is reserved for patients that have failed catheter ablation or are scheduled to undergo surgery for another indication.

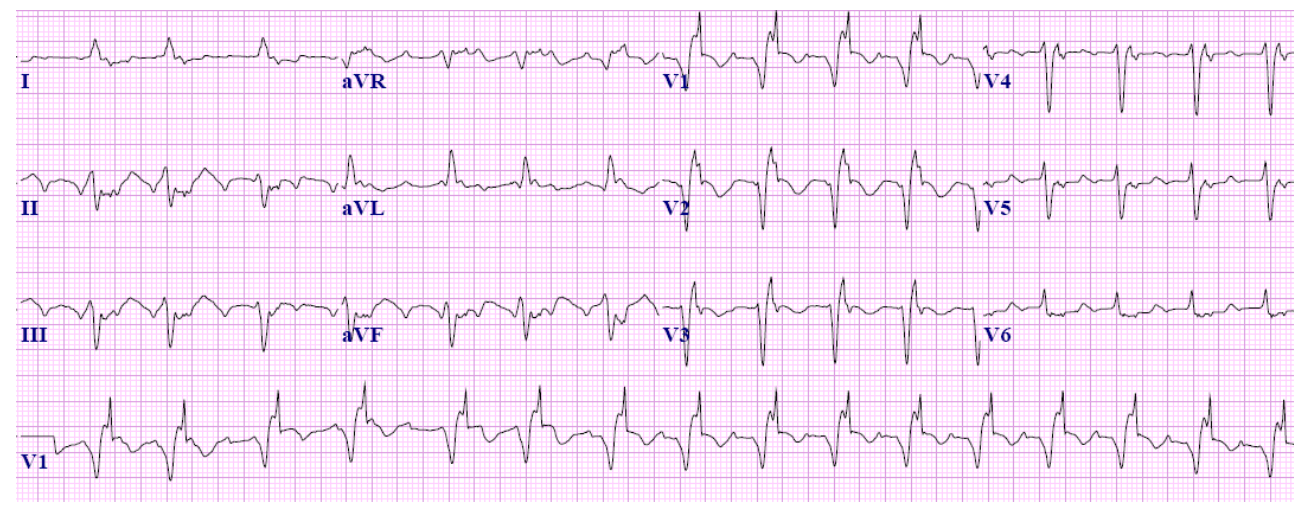

Fig. 11. Intra-atrial re-entrant tachycardia

\subsection{Atrio-Ventricular Reciprocating Tachycardia}

6.1.1 Definition: Atrio-ventricular reciprocating tachycardia (AVRT) is a re-entrant tachycardia with an accessory electrical connection between the atrial and ventricular myocardium in addition to the AV node (Blaufox and Saul 2001). The accessory pathway (AP) can be "concealed" with only retrograde conduction, "manifest" with antegrade or bidirectional conduction. The manifestation of antegrade conduction is presence of preexcitation with short PR on the electrocardiogram during sinus rhythm suggestive of WolffParkinson-White (WPW) syndrome. Orthodromic AVRT employs prograde conduction through the AV node and bundle of His with retrograde conduction through the AP. Antidromic AVRT employs AP for atrio-ventricular conduction with retrograde conduction through the bundle of His and AV node.

6.1.2 Incidence: AVRT is the most common cause of supraventricular tachycardia in children without prior cardiac surgery or neuromuscular disease, comprising 73 to 85 percent (Ko et al. 1992; Etheridge and Judd 1999). It is more common in infancy compared to AVNRT, the incidence of which increases in older children (Ko et al. 1992). Accessory pathways are associated with congenital heart defects in $6-26 \%$ of the children. The common heart defects associated with accessory pathways are Ebstein's anomaly of the tricuspid valve, ventricular inversion (L-TGA), hypertrophic cardiomyopathy, and mitral valve prolapse (Van Hare 1999). 
6.1.3 Mechanism: As the name suggests, AVRT has a reentrant mechanism. The reentrant mechanism is characterized by an accessory pathway in the left or right atrio-ventricular grooves, i.e. extranodal pathway. Atriofascicular and nodofascicular (Mahaim), and atrionodal (James) AP are other variants. The left free wall is the most common site of the $\mathrm{AP}$, followed by posteroseptal and right free wall AP (Calkins et al. 1999). Even in patients with manifest $\mathrm{AP}$, orthodromic narrow complex reciprocating tachycardia is still the most common manifestation.

6.1.4 Natural history: In patients with WPW syndrome with onset less than 2 months of age, SVT disappeared in $93 \%$ and reappeared in $31 \%$ at an average age of 8 years (Perry and Garson Jr. 1990). In patients with onset after 5 years of age, seventy eight percent had persistence at 7 years of follow-up. Multiple and right-sided AP were more frequent in patients with congenital heart disease (Perry and Garson Jr. 1990). Congenital heart defects are present in $20-37 \%$ of patients with WPW (Deal et al. 1985; Perry and Garson Jr. 1990). Patients with WPW syndrome are also prone to develop atrial fibrillation with increased risk of sudden death (Hare 1999).

6.1.5 Clinical presentation: Symptoms are usually paroxysmal but may be incessant. Symptoms may occur with or without triggers, including activity and exertion. Location, rate, duration of tachycardia, and the type of AP very often determine the nature of symptoms. Infants tolerate higher rates of tachycardia compared to adolescents and adults. Patients perceive sensed tachycardia with or without associated symptoms including dizziness, lightheadedness, and mild chest discomfort. Heart failure symptoms with orthopnea, paroxysmal nocturnal dyspnea, fatigue, tachypnea, diaphoresis are often present in those with persistent tachycardia (Van Hare 1999). Sudden death is attributed to the presence of atrial fibrillation with rapid ventricular response across the accessory pathway (Van Hare 1999). In orthodromic AVRT, shortness of breath, fatigue, and dizziness are common; syncope is less common (Blaufox and Saul 2001). In antidromic AVRT, dizziness, syncope, and tendency towards unstable ventricular rhythm are more common than orthodromic AVRT (Blaufox and Saul 2001).

6.1.6 ECG: ECG findings are determined by presence of tachycardia, normal sinus rhythm, and type of accessory pathway. In normal sinus rhythm, WPW syndrome is evident by the presence of pre-excitation (delta wave) with short PR interval. The pre-excited complex is a fusion complex resulting from initial pre-excitation of ventricular myocardium adjacent to the AP giving rise to the delta wave, followed by fusion of depolarization of the remainder of the ventricular myocardium by normal conduction. In orthodromic AVRT, the QRS complexes are narrow due to normal prograde conduction through the AV node and bundle of His and retrograde conduction via the AP (figure 12). Orthodromic AVRT have RP interval shorter than the PR interval with a P-wave on the upstroke of the T-wave during tachycardia, which differentiates it from AVNRT (Van Hare 1999). In antidromic AVRT, the QRS complexes are wide due to antegrade conduction down the AP and retrograde conduction across the AV node or another AP. AV block terminates the tachycardia due to the role of AV node in the tachycardia. WPW is characterized by wide QRS complexes with short PR interval. Mahaim AP has pre-excitation with widened QRS complexes but normal PR interval and usually manifest as a wide complex tachycardia with left bundle branch block pattern. Though the terminology is obsolete, Lown-Ganong-Levine (LGL) syndrome has normal QRS complexes with short PR interval.

6.1.7 Work-up: Baseline ECG during sinus rhythm may show pre-excitation. Depending on the frequency of sensed tachycardia or other symptoms, a Holter monitor or a loop recorder can be used. Echocardiogram may be obtained to rule out structural heart defects. In 
patients with pre-excitation, exercise stress test to stratify the risk of sudden catastrophic event can be performed. Persistent pre-excitation at peak exercise may suggest a short refractory period of the accessory fiber with a higher risk for sudden catastrophic event (Blaufox and Saul 2001). To differentiate between narrow complex tachycardias, including concealed pathways, AVNRT, and atrial tachycardias, an EP study is required. An EP study will also assist in differentiating antidromic AVRT from other wide-complex tachycardias.

6.1.8 Management and Prognosis: Acute management in patients with significant hemodynamic compromise requires synchronized DC cardioversion. Adenosine administration is effective and esmolol may be used. Flecainide and procainamide are recommended in pre-excited SVT (Blomstrom-Lundqvist 2003). Digoxin and calcium channel blockers are contra-indicated in patients with WPW syndrome. In stable patients, vagal maneuvers may be effective in terminating the arrhythmia. Calcium channel blockers are contraindicated in infants. Catheter ablation is a class I indication for managing AVRT (Blomstrom-Lundqvist 2003). Left free wall $\mathrm{AP}$ was predictive of ablation success while right free wall, posteroseptal, septal, and multiple APs were predictive of recurrence (Calkins et al. 1999). Medical therapy includes beta blockers to block AV node conduction and decrease premature ectopic beats, and class IA, IC, and III antiarrhythmics (Triedman 2001).

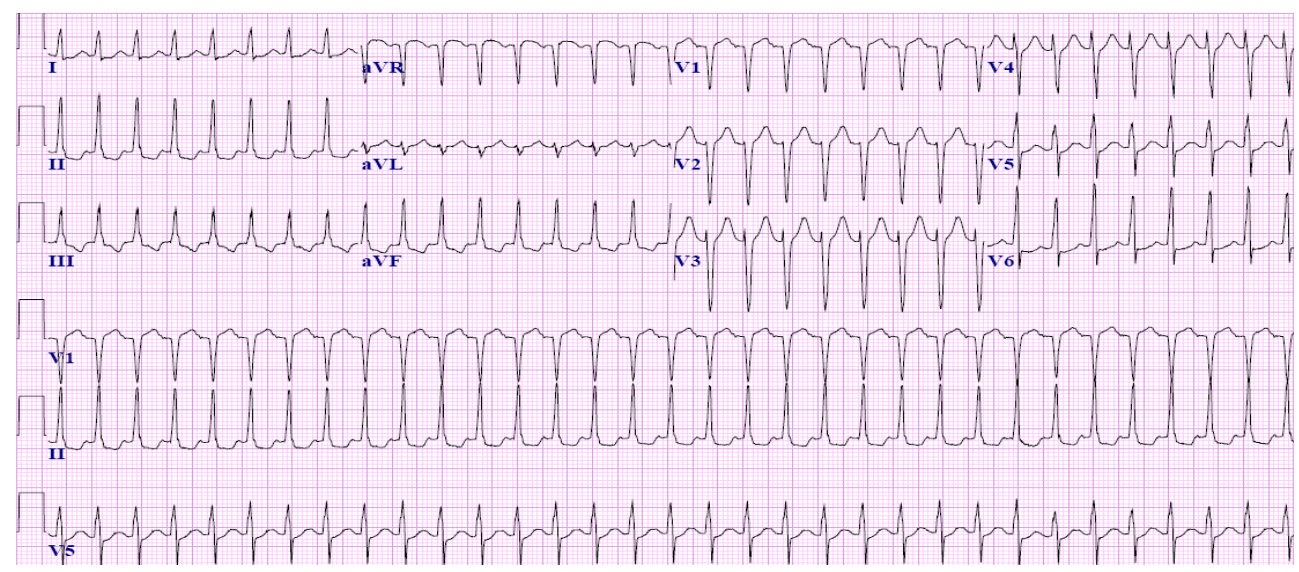

Fig. 12. Orthodromic AV reciprocating tachycardia

\subsection{Atrio-Ventricular Node Reentry Tachycardia}

6.2.1 Definition: Atrio-ventricular node reentry tachycardia (AVNRT) is a re-entry tachycardia that is dependent on existence of dual discrete pathways within or in proximity of the AV node, one with slow conduction and short effective refractory period and the other with fast conduction and long refractory period (Zimmerman 2001; Lockwood et al. 2004). The typical ventricular rate in children ranges from 120 to 280 beats/minute (Zimmerman 2001). AVNRT is often associated with an accessory pathway.

6.2.2 Incidence: AVNRT is the most common SVT in adults but consists of $13 \%$ of SVT in children without underlying heart disease and rarely in children under two years of age (Ko et al. 1992). In infants, $15 \%$ of SVT was due to AVNRT (Etheridge and Judd 1999).

6.2.3 Mechanism: Dual AV node physiology is present in 33-35\% of the children without clinical or inducible AVNRT in congenital or acquired heart disease (Casta et al. 1980). The 
"slow-fast" or typical form of the AVNRT is the most common form. A premature atrial or junctional beat blocks the fast pathway with subsequent antegrade conduction through the slow pathway. If the fast pathway recovers as the impulse conducts through the slow pathway, the substrate for reentry is present. In the less common "fast-slow" or atypical form of AVNRT, the premature atrial impulse is blocked in the slow pathway and is conducted antegrade along the fast pathway. If the slow pathway recovers, a reentry substrate is present; antegrade conduction down the fast pathway and retrograde conduction up the slow pathway is present (Zimmerman 2001). The least common form is the "slow-slow" dual node physiology. There is retrograde atrial activation with early atrial activation in the low right atrium, followed by left and remaining right atrial activation (Olgin and Zipes 2005a).

6.2.4 Natural history: AVNRT is not life-threatening and patients with minimal symptoms do well without therapy. Female patients are more symptomatic and tend to present with syncope (Drago et al. 2006).

6.2.5 Clinical presentation: Symptoms include palpitations, chest pain, heart failure, and shock, and rarely syncope, dependent on the duration of the AVNRT. Tachycardia is paroxysmal.

6.2.6 ECG: AVNRT is a regular narrow QRS complex tachycardia with abrupt onset and termination. The QRS complex may appear wider in case of baseline bundle branch block or rate dependent aberrant conduction. There is no pre-excitation appreciated during normal sinus rhythm in sole AVNRT. In slow-fast AVNRT, retrograde P-waves are not appreciated and are located within the terminal part of the QRS complexes. In fast-slow AVNRT, the P waves have a superior axis with RP interval longer than PR interval.

6.2.7 Work-up: An electrophysiology study is indicated to definitively establish the diagnosis of AVNRT and dual AV node physiology.

6.2.8 Management and Prognosis: Management of AVNRT is based on the frequency and the symptoms during the episodes. In those with infrequent, asymptomatic episodes, no treatment is necessary. Paroxysmal episodes may be terminated with Valsalva maneuver or carotid sinus massage. In acute decompensation, DC synchronized cardioversion, adenosine, IV beta blockers or digoxin can be employed (Zimmerman 2001). In patients with infrequent episodes desiring complete control, ablation is recommended. Patients with recurrent AVNRT refractory to beta blockers and calcium channel blockers, flecanide and sotalol are class IIA recommendations. In recurrent, symptomatic patients with significant side effects of medications and those with poorly tolerated AVNRT, catheter ablation of the slow AV nodal pathway is suggested (Blomstrom-Lundqvist 2003).

\section{Junctional Tachy-arrhythmias}

\subsection{Junctional Ectopic Tachycardia}

7.1.1 Definition: Junctional ectopic tachycardia (JET), also called junctional automatic tachycardia, is an incessant tachycardia characterized by rapid heart rate with the focus of abnormal automaticity in the junction. Ventricular rates in patients with JET ranges from 140-370 beats/minute (Villain et al. 1990).

7.1.2 Incidence: Incidence of JET occurs in two cohorts: neonatal and post-operative patients. Congenital JET occurs in the first six months of life and a family history is usually present (Sarubbi et al. 2002). Overall, JET occurs in 1\% of congenital heart repairs (Walsh 2001a). JET occurred in $22 \%$ of Tetralogy of Fallot (TOF) repairs, $10 \%$ of atrio-ventricular septal defect 
repairs, and 3.7\% of VSD repairs (Dodge-Khatami et al. 2002). Predictors of post-operative JET were younger age (Hoffman et al. 2002), lower body weight (Rekawek et al. 2007), resection of muscle bundles, higher bypass temperatures, and relief of right ventricular outflow tract obstruction through the right atrium (Dodge-Khatami et al. 2002).

7.1.3 Mechanism: Although the mechanism of JET is unclear, abnormal (enhanced) automaticity of the junction has been put forth. In post-operative congenital heart disease patients, it is hypothesized that direct trauma or infiltrative hemorrhage of the conduction system leads to enhanced automaticity of the His bundle (Dodge-Khatami et al. 2002).

7.1.4 Natural history: Congenital JET is associated with high mortality, most of which is attributed to sudden cardiac death (Villain et al. 1990). Concurrent arrhythmias may co-exist with JET, such as complete heart block, ventricular tachycardia, and persistent junctional reciprocating tachycardia. Post-operative JET is a transient state lasting on average 36 hours (Walsh 2001a) and is associated with higher mortality and longer intensive care unit (ICU) stay (Andreasen et al. 2008).

7.1.5 Clinical presentation: Congenital JET presents with cardiomegaly with concomitant heart failure in more than 50 percent, death in 35 percent (Villain et al. 1990), hydrops fetalis, and echocardiographic evidence of left ventricular dysfunction. In post-operative JET, the decreased ventricular filling time leads to cardiovascular compromise.

7.1.6 ECG: ECG criteria include 1) QRS morphology similar to that of sinus rhythm, 2) rapid ventricular rate, and 3) dissociated sinus rhythm with atrial rate often less than ventricular rate or retrograde 1:1 conduction (Walsh 2001a). If variability exists in R-R interval, appropriately timed P-waves may conduct to the ventricular tissue (Figure 13).

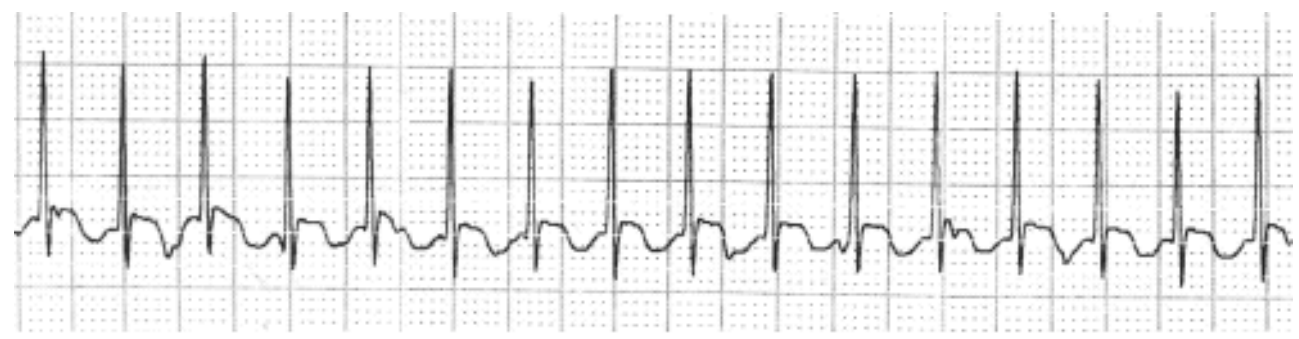

Fig. 13. Junctional ectopic tachycardia

7.1.7 Work-up: An electrophysiology study is generally not necessary in patients with JET, unless ablation is being considered for medication-refractory symptoms (Walsh 2001a). In cases of 1:1 retrograde conduction across the AV node, adenosine can clarify the underlying rhythm by blocking the AV node.

7.1.8 Management and Prognosis: Management of post-operative JET includes achievement of AV synchrony and rate control. Atrial pacing above the rate of JET may establish rate control (Walsh 2001a). Post-operative JET is generally responsive to intravenous amiodarone, a class III antiarrhythmic (Shah and Rhodes 1998). Mean length of time for termination of postoperative JET after loading and infusion of amiodarone was 4.5 hours with majority achieving control within 12 hours (Plumpton et al. 2005). Decreasing the body core temperature to 32-34 ${ }^{\circ} \mathrm{C}$, decreasing the sympathomimetic catecholamine levels, optimizing volume, electrolytes and hemoglobin contribute to decreased ventricular rate by decreasing the automaticity (Cabrera et al. 2002). Magnesium supplementation has been shown to be beneficial in 
prevention of post-operative JET (Dorman et al. 2000). JET is unresponsive to DC cardioversion, overdrive pacing, and programmed atrial or ventricular stimulation (Sarubbi et al. 2003). Pharmacologic treatment of congenital JET with monotherapy is less successful than combined therapy with amiodarone, digoxin or class IA medication (Sarubbi et al. 2002). Patients with drug-refractory congenital JET should undergo catheter ablation with maintenance of the normal atrio-ventricular conduction (Walsh 2001a). A pacemaker is recommended in those children with evidence of impaired conduction with atrial stimulation, sinus dysfunction, or spontaneous AV block on ECG or Holter monitor (Walsh 2001a).

\subsection{Persistent Junctional Reciprocating Tachycardia}

7.2.1 Definition: Persistent or permanent junctional reciprocating tachycardia (PJRT) is an incessant tachycardia characterized by prograde conduction through the AV node and retrograde conduction through a slow accessory pathway located in the posteroseptal (74\%) region with decremental conduction properties (Critelli, G. et al. 1984; Lindinger, A. et al. 1998; Vaksmann, G. et al. 2006). PJRT is a narrow complex tachycardia with rates ranging from 120-250 beats/min and infants having higher tachycardia rates than children (Dorostkar et al. 1999).

7.2.2 Incidence: PJRT consists of $1 \%$ to $6 \%$ of all supraventricular tachycardia (Dorostkar et al. 1999; Blaufox and Saul 2001). It can present in infancy and into early childhood.

7.2.3 Mechanism: PJRT has a reentrant mechanism with accessory pathways which are mostly isolated and in patients without congenital heart disease (Vaksmann, G et al. 2006).

7.2.4 Natural history: In a recent study by Vaksmann et al (2006), PJRT resolved spontaneously in 22 percent (Vaksmann, G et al. 2006). Long term persistence of PJRT can lead to tachycardia-mediated cardiomyopathy, which resolves with appropriate rate control (Lindinger, A et al. 1998; Noe et al. 2002). Uncontrolled PJRT may lead to death.

7.2.5 Clinical presentation: Patients often are asymptomatic in childhood. Symptomatic patients present with intermittent palpitations, exercise intolerance, and syncope (Dorostkar et al. 1999).

7.2.6 ECG: ECG shows retrograde P-wave in leads II, III, aVF, and left lateral leads, and R-P interval longer than P-R interval (Dorostkar et al. 1999). The long R-P interval is attributed to the slow retrograde conduction. In sinus rhythm, there is no delta wave and P-R interval is normal (Critelli, G et al. 1984). QRS complexes are narrow.

7.2.7 Work-up: PJRT is likely under-diagnosed in childhood and patients present with heart failure with associated ventricular dysfunction. An ECG and Holter monitor should be considered. An echocardiogram should be performed to assess the cardiac function and structure and serial echocardiograms are necessary in those with depressed LV function. An electrophysiology study and catheter ablation may be performed in refractory PJRT associated with decreased ventricular function (Dorostkar et al. 1999).

7.2.8 Management and Prognosis: Many patients with stable infrequent episodes and slow tachycardia may not require medical therapy (Lindinger, A et al. 1998). In pediatric patients, amiodarone and verapamil alone had success rates of 84-94\% (Vaksmann, $\mathrm{G}$ et al. 2006). Digoxin alone had a success rate of $50 \%$. Hence, medical therapy with anti-arrhythmic drugs should be advocated prior to consideration of catheter ablation (Drago et al. 2001). Pediatric and adult patients with medication-refractory incessant PJRT should undergo direct current catheter ablation, with good safety and efficacy (Aguinaga et al. 1998). 


\subsection{Ventricular Tachycardia}

8.1.1 Definition: Ventricular tachycardia (VT) is a tachycardia with rates greater than 120 beats per minute and originating within the ventricles or the lower conduction system with ventriculo-atrial dissociation. Non-sustained VT is defined as a three or more consecutive beats of ventricular origin that terminate spontaneously with no hemodynamic compromise and lasting less than 30 seconds. Sustained VT persists longer than 30 seconds or requires medical or electrical intervention to terminate the tachycardia (AHA 2007).

8.1.2 Incidence: In healthy teenage boys, short episodes of ventricular tachycardia were present in 3\% (Dickinson and Scott 1984). Incidence of VT is higher in patients with congenital heart disease. Approximately $30 \%$ of post-operative tetralogy of Fallot patients had sustained monomorphic VT and $4.4 \%$ had polymorphic VT, which contributes to $2 \%$ annual risk of sudden cardiac death (Khairy et al. 2004; Walsh, E. and Cecchin, F. 2007). VT originates in the RVOT in $60 \%-80 \%$ of structurally normal hearts (Lerman et al. 2004). Idiopathic VT is also associated with aortic valve disease, corrected transposition of the great arteries, Ebstein's anomaly of the tricuspid valve, Eisenmenger's syndrome, long QT syndrome, unrepaired tetralogy of Fallot, arrhythmogenic right ventricular dysplasia, and myocardial infarction (Callans, D. and Josephson, M. 2004; Fontaine et al. 2004; Walsh, E. P. and Cecchin, F. 2007).

8.1.3 Mechanism: Ventricular tachycardia characterized by a re-entry mechanism can be initiated and terminated with programmed stimuli (Callans, D and Josephson, ME 2004). VT may also be secondary to automaticity or triggered activity.

8.1.4 Natural history: Post-operative congenital heart disease patients are at high risk of VT and sudden cardiac death. In patients with normal hearts, younger children (90\%) and older populations (50\%-70\%) have resolution of VT within 2 to 10 years (Alexander 2001b). However, symptomatic younger patients had worse prognosis (Davis et al. 1996). In patients with normal intracardiac anatomy, only $50 \%$ have an actual diagnosis such as hypertrophic cardiomyopathy, dilated cardiomyopathy, myocarditis, myocardial hamartoma, and metabolic disease (Davis et al. 1996). Ventricular tachycardia is present in $50 \%-60 \%$ of patients with dilated cardiomyopathy and responsible for $8 \%-50 \%$ of deaths (Galvin and Ruskin 2004).

8.1.5 Clinical presentation: Clinical presentation is variable (Davis et al. 1996). Symptoms may range from palpitations, light headedness, syncope, shortness of breath, neck fullness, and chest pain to death (Silka and Garson Jr. 1999).

8.1.6 ECG: Monomorphic VT is a wide complex tachycardia with same QRS morphology, which suggests a single re-entrant focus (figure 14). Polymorphic VT is a wide complex tachycardia with variable QRS morphology. Torsades de pointe is a form of polymorphic VT and associated with long QT syndrome and variable electrical activation. (Figure 15)

8.1.7 Work-up: Obtain a thorough personal and family history including history of sudden cardiac death. An ECG of the arrhythmia is useful but very difficult to capture very often; however, a resting ECG is a class 1 recommendation (Zipes and Camm 2006). Check electrolytes, in particular hypokalemia, hyperkalemia, and hypomagnesemia. In patients with recurrent syncope, a holter monitor or event recorder is indicated. An echocardiogram is necessary to identify structural heart disease and evaluate cardiac function in those at risk for sudden cardiac death such as dilated, hypertrophic, and arrhythmogenic RV dysplasia, myocarditis, and inherited disorders (Zipes and Camm 2006). An exercise stress test is indicated in all individuals, regardless of age, known or suspected to have exercise-induced ventricular tachycardia. An electrophysiology study is warranted in patients with 
congenital/structural heart disease, those with unknown etiology of VT, and those with syncope of unknown etiology with LV dysfunction (Zipes and Camm 2006).

8.1.8 Management and Prognosis: All wide complex tachycardias need to be treated like ventricular tachycardia unless proven otherwise. A-V dissociation, superior QRS axis and positive or negative concordance of QRS complexes in the precordial leads suggest ventricular origin. In patients with normal hearts, episodes of non-sustained VT has good prognosis. Avoid medications that prolong QT interval and correct electrolyte abnormalities. In patients with sustained VT with hemodynamic compromise, direct current (DC) cardioversion is indicated (Zipes and Camm 2006). In stable monomorphic VT, IV procainamide or amiodarone, and/or transvenous catheter pace termination maybe employed. In patients with polymorphic VT, DC cardioversion, IV amiodarone and beta blockers maybe employed. Internal cardiac defibrillator is indicated in patients with congenital heart disease who have survived cardiac arrest, patients with severely impaired ventricular function, and those on chronic optimal medical therapy with a life expectancy of at least one year (Zipes and Camm 2006).

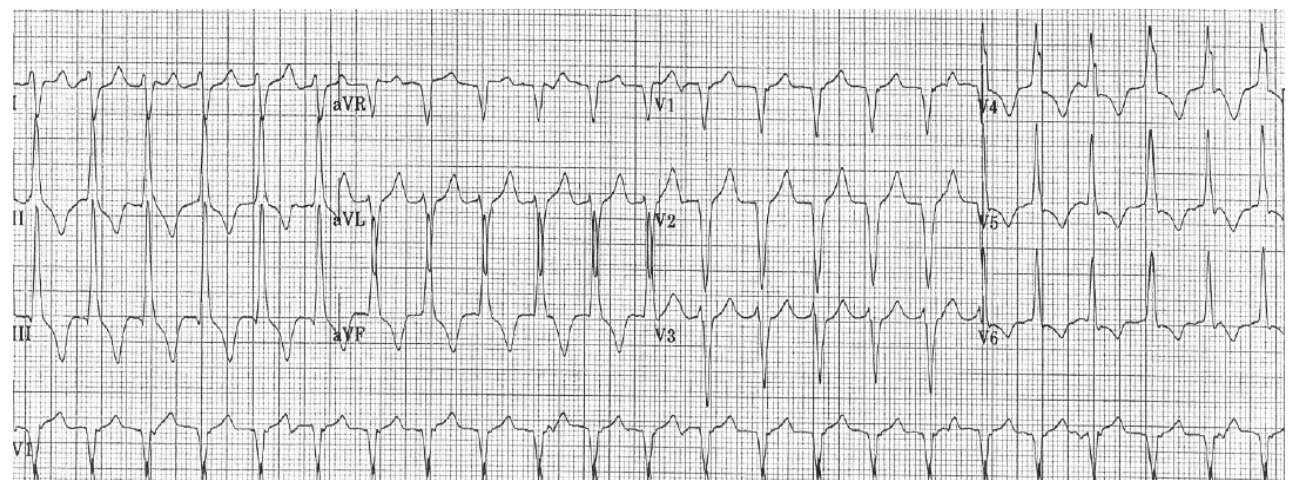

Fig. 14. Ventricular tachycardia

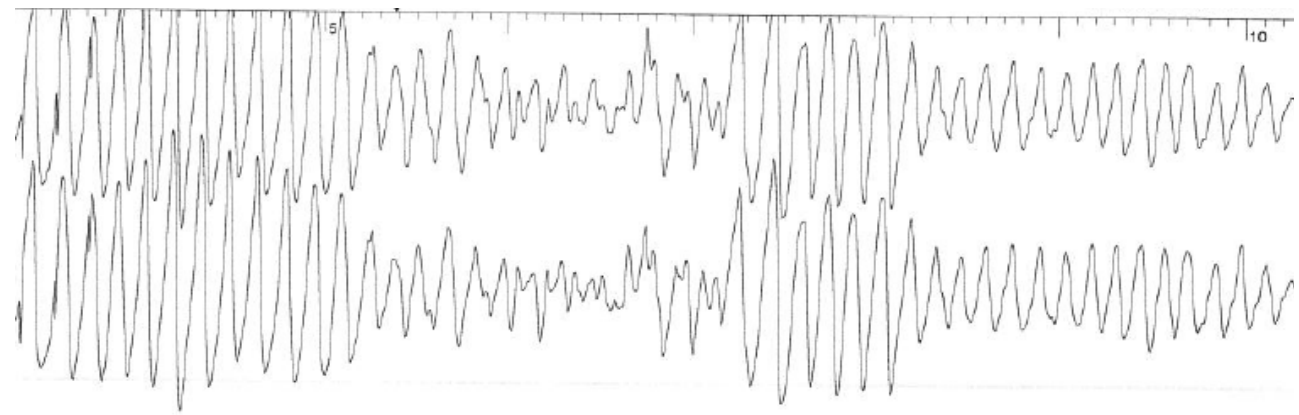

Fig. 15. Torsades de pointe 


\section{References}

Aellig, N. C.C. Balmer, et al. (2007). "Long-term follow-up after pacemaker implantation in neonates and infants." Ann Thorac Surg 83(4): 1420-3.

Aguinaga, L.J. Primo, et al. (1998). "Long-Term Follow-Up in Patients with the Permanent Form of Junctional Reciprocating Tachycardia Treated with Radiofrequency Ablation." Pacing and Clinical Electrophysiology 21(11): 2073-2078.

Ajisaka, H.T. Hiraki, et al. (1997). "Direct Conversion of Atrial Flutter to Sinus Rhythm with Low-output, Short-duration Transesophageal Atrial Pacing." Clinical Cardiology 20(9): 762-6.

Alexander, M. (2001b). Ventricular Arrhythmias in Children and Young Adults. Cardiac Arrhythmias in Children and Young Adults with Congenital Heart Disease. E. Walsh, J. Saulet al. Philadelphia, PA, Lippincott Williams \& Wilkins: 201-234.

Alison, J. F.J. A. Yeung-Lai-Wah, et al. (1995). "Characterization of junctional rhythm after atrioventricular node ablation." Circulation 91(1): 84-90.

Andreasen, J., S. Johnsen, et al. (2008). "Junctional Ectopic Tachycardia after Surgery for Congenital Heart Disease in Children." Intersive Care Medicine 34(5): 895-902.

Andrew, P. and A. Montenero (2007). "Atrial Flutter: A Focus on Treatment Options for a Common Supraventricular Tachyarrhythmia." Journal of Cardiovascular Medicine 8: 558-567.

Attina, D. A.F. Mori, et al. (1987). "Long-term follow-up in children without heart disease with ventricular premature beats." Eur Heart J 8 Suppl D: 21-3.

Beaufort-Krol, G. C.M. J. Schasfoort-van Leeuwen, et al. (2007). "Longitudinal echocardiographic follow-up in children with congenital complete atrioventricular block." Pacing Clin Electrophysiol 30(11): 1339-43.

Benditt, D. G.G. J. Klein, et al. (1984). "Enhanced atrioventricular nodal conduction in man: electrophysiologic effects of pharmacologic autonomic blockade." Circulation 69(6): 1088-95.

Blaufox, A. and J. Saul (2001). Accessory-Pathway-Mediated Tachycardias. Cardiac Arrhythmias in Children and Young Adults with Congenital Heart Disease. E. Walsh, J. Saulet al. Philadelphia, PA, Lippincott Williams \& Wilkins: 173-199.

Blomstrom-Lundqvist, C.M. M. Scheinman, et al. (2003). "ACC/AHA/ESC guidelines for the management of patients with supraventricular arrhythmias--executive summary. a report of the American college of cardiology/American heart association task force on practice guidelines and the European society of cardiology committee for practice guidelines (writing committee to develop guidelines for the management of patients with supraventricular arrhythmias) developed in collaboration with NASPE-Heart Rhythm Society." J Am Coll Cardiol 42(8): 1493531.

Brodsky, M.D. Wu, et al. (1977). "Arrhythmias documented by 24 hour continuous electrocardiographic monitoring in 50 male medical students without apparent heart disease." Am J Cardiol 39(3): 390-5.

Cabrera, D.D. Rodrigo Carbonero, et al. (2002). "The Treatment of Post-operative Junctional Ectopic Tachycardia." Anales Espanoles de Pediatria 56(6): 505-9.

Calkins, H.P. Yong, et al. (1999). "Catheter Ablation of Accessory Pathways, Atrioventricular Nodal Reentrant Tachycardia, and the Atrioventricular Junction: Final Results of a Prospective, Multicenter Clinical Trial." Circulation 99(2): 262-70. 
Callans, D. and M. Josephson (2004). Ventricular tachycardia in patients with coronary artery disease. Cardiac Electrophysiology: From cell to bedside. D. P. Zipes and J. Jalife. Philadelphia, PA: 569-574.

Case, C. L. (1999). "Diagnosis and treatment of pediatric arrhythmias." Pediatr Clin North Am 46(2): 347-54

Casta, A.G. Wolff, et al. (1980). "Dual Atrioventricular Nodal Pathways: A Benign Finding in Arrhythmia-free Children with Heart Disease." American Journal of Cardiology 46(6): 1013-8.

Chandar, J. S.G. S. Wolff, et al. (1990). "Ventricular arrhythmias in postoperative tetralogy of Fallot." Am J Cardiol 65(9): 655-61.

Cheitlin, M. D.W. F. Armstrong, et al. (2003). "ACC/AHA/ASE 2003 guideline update for the clinical application of echocardiography--summary article: a report of the American College of Cardiology/American Heart Association Task Force on Practice Guidelines (ACC/AHA/ASE Committee to Update the 1997 Guidelines for the Clinical Application of Echocardiography)." J Am Coll Cardiol 42(5): 954-70.

Chiale, P.H. Garro, et al. (2006). "Inappropriate Sinus Tachycardia may be Related to an Immunologic Disorder Involving Cardiac Beta Adrenergic Receptors." Heart Rhythm 3(10): 1182-6.

Chiang, B. N.L. V. Perlman, et al. (1969). "Relationship of premature systoles to coronary heart disease and sudden death in the Tecumseh epidemiologic study." Ann Intern Med 70(6): 1159-66.

Coker, R.A. Koziell, et al. (1984). "Does the sympathetic nervous system influence sinus arrhythmia in man? Evidence from combined autonomic blockade." J Physiol 356: 459-64.

Critelli, G.J. J. Gallagher, et al. (1984). "Anatomic and electrophysiologic substrate of the permanent form of junctional reciprocating tachycardia." J Am Coll Cardiol 4(3): 601-10.

Davis, A.R. Gow, et al. (1996). "Clinical Spectrum, Therapeutic Management, and Follow-up of Ventricular Tachycardia in Infants and Young Children." American Heart Journal 131: 186-91.

Deal, B.J. Keane, et al. (1985). "Wolff-Parkinson-White Syndrome and Supraventricular Tachycardia During Infancy: Management and Follow-up." Journal of The American College of Cardiology 5(1): 130-5.

Dewey, R. C., M. A. Capeless, et al. (1987). "Use of ambulatory electrocardiographic monitoring to identify high-risk patients with congenital complete heart block." N Engl J Med 316(14): 835-9.

Dhingra, R. C.P. Denes, et al. (1974). "The significance of second degree atrioventricular block and bundle branch block. Observations regarding site and type of block." Circulation 49(4): 638-46.

Dickinson, D. and O. Scott (1984). "Ambulatory Electrographic Monitoring in 100 Healthy Teenage Boys." British Heart Journal 51(2): 179-83.

Dodge-Khatami, A.O. Miller, et al. (2002). "Surgical Substrates of Postoperative Junctional Ectopic Tachycardia in Congenital Heart Defects." Journal of Thoracic and Cardiovascular Surgery 123(4): 624-630. 
Dorman, B.R. Sade, et al. (2000). "Magnesium Supplementation in the Prevention of Arrhythmias in Pediatric Patients Undergoing Surgery for Congenital Heart Defects." American Heart Journal 139(3): 522-8.

Dorostkar, P.M. Silka, et al. (1999). "Clinical Course of Persistent Junctional Reciprocating Tachycardia." Journal of The American College of Cardiology 33: 366-75.

Drago, F.G. Grutter, et al. (2006). "Atrioventricular Nodal Reentrant Tachycardia in Children." Pediatric Cardiology 27(4): 454-59.

Drago, F.M. Silvetti, et al. (2001). "Permanent Juncational Reciprocating Tachycardia in Infants and Children: Effectiveness of Medical and Non-Medical Treatment." Italian Heart Journal 2(6): 456-61.

Dubin, D. (2000). Rapid Interpretation of EKGs. Fort Meyers, COVER Publishing Company, Pages.

Duster, M. C.M. T. Bink-Boelkens, et al. (1985). "Long-term follow-up of dysrhythmias following the Mustard procedure." Am Heart J 109(6): 1323-6.

Espinola-Zavaleta, N.M. E. Soto, et al. (2006). "Non-compacted cardiomyopathy: clinicalechocardiographic study." Cardiovasc Ultrasound 4: 35.

Etheridge, S. and V. Judd (1999). "Supraventricular Tachycardia in Infancy: Evaluation, Management, and Follow-up." Archives of Pediatric Adolescent Medicine 153(3): 267-71.

Ferrer, P. (1977). Arrhythmias in the neonate, infant, and child. Arrhythmias in the Neonate. N. Roberts and H. Gelband. New York, Appleton-Century-Crofts: 265-316.

Fisch, C. and S. B. Knoebel (1970). "Junctional rhythms." Prog Cardiovasc Dis 13(2): 141-58.

Fishberger, S. (2001). Sinus Node Dysfunction. Cardiac Arrhythmias in Children and Young Adults with Congenital Heart Disease. E. Walsh, J. Saulet al. Philadelphia, Lippincott Williams \& Wilkins: 271-283.

Fogoros, R. (2006). The Electrophysiology Study in the Evaluation of the SA Node, AV Node, and His-Purkinje System. Malden, MA, Blackwell Publishing, Inc., Pages.

Fontaine, G.P. Forres, et al. (2004). Ventricular Tachycardia in Arrhythmogenic Right Ventricular Cardiomyopathies. Cardiac Electrophysiology: From cell to bedside. D. P. Zipes and J. Jallife. Philadelphia, PA, W.B.Saunders: 588-600.

Frolkis, J. P.C. E. Pothier, et al. (2003). "Frequent ventricular ectopy after exercise as a predictor of death." N Engl J Med 348(9): 781-90.

Galvin, J. and J. Ruskin, Eds. (2004). Ventricular Tachycardia in Patients with Dilated Cardiomyopathy. Philadelphia, PA, Saunders: Pages.

Gamble, Y. D., W. P. Lutin, et al. (2007). "Non-sinus bradyarrhythmias in very low birth weight infants." J Perinatol 27(1): 65-7.

Garan, H. (2008). "Atypical Atrial Flutter." Heart Rhythm 5: 618-621.

Garson Jr., A.M. Bink-Boelkens, et al. (1985). "Atrial Flutter in the Young: a Collaborative Study of 380 cases." Journal of The American College of Cardiology 6(4): 871-8.

Garson Jr., A. and P. Gillette (1981). "Electrophysiologic Studies of Supraventricular Tachycardia in Children. I. Clinical-electrophysiologic Correlations." American Heart Journal 102(2): 233-250.

Gelatt, M.R. M. Hamilton, et al. (1994). "Risk factors for atrial tachyarrhythmias after the Fontan operation." J Am Coll Cardiol 24(7): 1735-41.

Gibbons, R. J.G. J. Balady, et al. (2002). "ACC/AHA 2002 guideline update for exercise testing: summary article: a report of the American College of Cardiology/American 
Heart Association Task Force on Practice Guidelines (Committee to Update the 1997 Exercise Testing Guidelines)." Circulation 106(14): 1883-92.

Gomes, A., R. Hariman, et al. (1985). "Sustained Symptomatic Sinus Node Reentrant Tachycardia: Incidence, Clinical Significance, Electrophysiologic Observations and the Effects of Antiarrhythmic Agents." Journal of The American College of Cardiology 5: 45-47.

Goya, M.Y. Iesaka, et al. (1999). "Radiofrequency Catheter Ablation for Sinoatrial Node Reentrant Tachycardia." Japanese Circulation Journal 63: 177-83.

Granada, J.W. Uribe, et al. (2000). "Incidence and Predictors of Atrial Flutter in the General Population." Journal of The American College of Cardiology 36(7): 2242-6.

Gregaratos, G. (2002). "ACC/AHA/NASPE 2002 Guideline Update for Implantation of Cardiac Pacemakers and Antiarrhythmia Devices." Circulation.

Haines, D. and J. DiMarco (1990). "Sustained intraatrial reentrant tachycardia: clinical, electrocardiographic and electrophysiologic characteristics and long-term followup." Journal of The American College of Cardiology 15(6): 1345-54.

Hare, G. V. (1999). Supraventicular Tachycardia. Clinical Pediatric Arrhythmias. P. G. A. G. Jr. Philadelphia, W.B.Saunders Company: 97-120.

Hiss, R. G. and L. E. Lamb (1962). "Electrocardiographic findings in 122,043 individuals." Circulation 25: 947-61.

Hoffman, B. F. and P. F. Cranefield (1964). "The Physiological Basis Of Cardiac Arrhythmias." Am J Med 37: 670-84.

Hoffman, T.D. Bush, et al. (2002). "Postoperative Junctional Ectopic Tachycardia in Children: Incidence, Risk Factors, and Treatment." Annals of Thoracic Surgery 74(5): 1607-11.

Hsieh, M. and S. Chen (2002). Catheter Ablation in Focal Tachycardia. Catheter Ablation of Arrhythmias. D. Zipes and M. Haissaguerre. Armonk, NY, Futura Publishing Co., Inc.: 185-204.

Huhta, J. C.J. D. Maloney, et al. (1983). "Complete atrioventricular block in patients with atrioventricular discordance." Circulation 67(6): 1374-7.

Jacquet, L.G. Ziady, et al. (1990). "Cardiac rhythm disturbances early after orthotopic heart transplantation: prevalence and clinical importance of the observed abnormalities." J Am Coll Cardiol 16(4): 832-7.

Jideus, L.M. Kesek, et al. (2006). "The role of premature atrial contractions as the main triggers of postoperative atrial fibrillation." J Electrocardiol 39(1): 48-54.

Karpawich, P. P.P. C. Gillette, et al. (1981). "Congenital complete atrioventricular block: clinical and electrophysiologic predictors of need for pacemaker insertion." Am J Cardiol 48(6): 1098-102.

Kastor, J. (1990). "Multifocal Atrial Tachycardia." New England Journal of Medicine 322: 1713.

Kaushal, P. and J. A. Taylor (2002). "Inter-relations among declines in arterial distensibility, baroreflex function and respiratory sinus arrhythmia." J Am Coll Cardiol 39(9): 1524-30.

Khairy, P.M. Landzberg, et al. (2004). "Value of Programmed Ventricular Stimulatino after Tetralogy of Fallot Repair: a Multicenter Study." Circulation 109(16): 1994-2000.

Kleinfeld, M. J. and B. H. Boal (1978). "Junctional escape rhythm in the sick sinus syndrome." Cardiology 63(4): 193-8. 
Ko, J.B. Deal, et al. (1992). "Supraventricular Tachycardia Mechanisms and their Age Distribution in Pediatric Patients." American Journal of Cardiology 69(12): 1028-32.

Kones, R., J. Phillips, et al. (1974). "Mechanism and Management of Chaotic Atrial Mechanism." Cardiology 59: 92.

Krahn, A.R. Yee, et al. (1995). "Inappropriate sinus tachycardia: evaluation and thearpy." Journal of Cardiovascular Electrophysiology 6(12): 1124-8.

Lerman, B.K. Stein, et al., Eds. (2004). Ventricular Tachycardia in Patients with Structurally Normal Hearts. Philadelphia, PA, Saunders: Pages.

Levine, S. A., H. Miller, et al. (1956). "Some clinical features of complete heart block." Circulation 13(6): 801-24.

Lin, D. and D. Callans (2004). Clinical Arrhythmias: Mechanisms, Features, and Management. Supraventricular Arrhythmias. Cardiac Electrophysiology From Cell to Bedside. D. Zipes and J. Jaliffe. Philadelphia, PA, Saunders: 479-484.

Lindinger, A.A. Heisel, et al. (1998). "Permanent junctional re-entry tachycardia. A multicentre long-term follow-up study in infants, children and young adults." Eur Heart J 19(6): 936-42.

Lockwood, D.K. Otomo, et al. (2004). Electrophysiologic Characteristics of Atrioventricular Nodal Reentrant Tachycardia: Implications for the Reentrant Circuits. Cardiac Electrophysiology From Cell to Bedside. D. Zipes and J. Jaliffe. Philadelphia, PA, Saunders: 537-557.

Lundstrom, U.C. Bull, et al. (1990). "The natural and "unnatural" history of congenitally corrected transposition." Am J Cardiol 65(18): 1222-9.

Martin, A. and J. Kugler (1999). Sinus Node Dysfunction. Clinical Pediatric Arrhythmias. P. Gillette and A. Garson, Jr. Philadelphia, W.B. Saunders Company: 51-62.

Michaelsson, M. and M. A. Engle (1972). "Congenital complete heart block: an international study of the natural history." Cardiovasc Clin 4(3): 85-101.

Morillo, C. and J. Guzman (2007). "Inappropriate sinus tachycardia: an update." Rev Esp Cardiol 60(Suppl 3): 10-14.

Mymin, D.F. A. Mathewson, et al. (1986). "The natural history of primary first-degree atrioventricular heart block." N Engl J Med 315(19): 1183-7.

Naheed, Z.J. Strasburger, et al. (1995). "Natural History and Management Strategies of Automatic Atrial Tachycardia in Children." American Journal of Cardiology 75(56): 405-7.

Narula, O. (1974). "A Mechanism for Supraventricular Tachycardia." Circulation 50: 1114-28.

Noe, P.V. Van Driel, et al. (2002). "Rapid Recovery of Cardiac Function after Catheter Ablation of Persistent Junctional Reciprocating Tachycardia in Children." Pacing and Clinical Electrophysiology 25(2): 191-4.

Olgin, J. and D. Zipes (2005a). Specific Arrhythmias: Diagnosis and Treatment. Braunwald's Heart Disease. D. ZipesP. Libbyet al. Philadelphia, PA, Elsevier Saunders. 1: 803-863.

Olgin, J. and D. Zipes (2005b). Specific Arrhythmias: Diagnosis and Treatment: Premature Ventricular Complexes. Braunwald's Heart Disease: A Textbook of Cardiovascular Medicine. D. ZipesE. Braunwaldet al. Philadelphia, Saunders: 838-841.

Packer, D.G. Bardy, et al. (1986). "Tachycardia-induced Cardiomyopathy: A Reversible Form of Left Ventricular Dysfunction." American Journal of Cardiology 57(8): 563-570.

Paridon, S. (2006). "Clinical stress testing in the pediatric age group. A statement from the American Heart Association Council on Cardiobascular Disease in the Young, 
Committee on Atherosclerosis, Hypertension, and Obesity in Youth." Circulation 113(15): 1905-20.

Perry, J. and A. Garson Jr. (1990). "Supraventricular Tachycardia due to Wolff-ParkinsonWhite Syndrome in Children: Early Disappearance and Late Recurrence." Journal of The American College of Cardiology 16(5): 1215-20.

Peuch, P., R. Groileau, et al. (1976). Incidence of different types of A-V block and their localization by His bundle recordings. The Conduction System of the Heart. H. J. Wellens, K. I. Lieet al. Leiden, Stenfert: 467.

Plumpton, K., R. Justo, et al. (2005). "Amiodarone for Post-operative Junctional Ectopic Tachycardia." Cariology in the Young 15(1): 13-8.

Poutiainen, A.M. Koistinen, et al. (1999). "Prevalence and natural course of ectopic atrial tachycardia." European Heart Journal 20(9): 694-700.

Randall, W. C., W. H. Wehrmacher, et al. (1981). "Hierarchy of supraventricular pacemakers." J Thorac Cardiovasc Surg 82(5): 797-800.

Rekawek, J.A. Kansy, et al. (2007). "Risk Factors for Cardiac Arrhythmias in Children with Congenital Heart Disease after Surgical Intervention in the Early Postoperative Period." Journal of Thoracic and Cardiovascular Surgery 133(4): 900-4.

Roberts-Thomson, K., P. Kistler, et al. (2006). "Focal Atrial Tachycardia I: Clinical Features, Diagnosis, Mechanisms, and Anatomic Location." Pacing and Clinical Electrophysiology 29(6): 643-652.

Roberts, N. K. and P. C. Gillette (1977). "Electrophysiologic study of the condition system in normal children." Pediatrics 60(6): 858-63.

Rosen, M. R.C. Fisch, et al. (1980). "Can accelerated atrioventricular junctional escape rhythms be explained by delayed afterdepolarizations?" Am J Cardiol 45(6): 127284.

Salim, M., C. Case, et al. (1995). "Chaotic Atrial Tachycardia in Children." American Heart Journal 129: 831-33.

Saoudi, N.F. Cosio, et al. (2001). "A Classification of Atrial Flutter and Regular Atrial Tachycardia According to Electrophysiological Mechanisms and Anatomica Bases." European Heart Journal 22: 1162-1182.

Sarubbi, B.B. Musto, et al. (2002). "Congenital Junctional Ectopic Tachycardia in Children and Adolescents: a 20 Year Experience Based Study." Heart 88: 188-190.

Sarubbi, B.P. Vergara, et al. (2003). "Congenital Junctional Ectopic Tachycardia: Presentation and Outcome." Indian Pacing and Electrophysiology Journal 3(3): 143-7.

Scott, O., G. J. Williams, et al. (1980). "Results of 24 hour ambulatory monitoring of electrocardiogram in 131 healthy boys aged 10 to 13 years." Br Heart J 44(3): 3048.

Sestito, A.M. Pardeo, et al. (2007). "Cardiac magnetic resonance of healthy children and young adults with frequent premature ventricular complexes." J Cardiovasc Med (Hagerstown) 8(9): 692-8.

Shah, M. and L. Rhodes (1998). "Management of Postoperative Arrhythmias and Junctional Ectopic Tachycardia." Seminars in Thoracic and Cardiovascular surgery. Pediatric Cardiac Surgery Annual 1: 91-102.

Shen, W. (2005). "How to Manage Patients with Inappropriate Sinus Tachycardia." Heart Rhythm 2(9): 1015-19. 
Shepard, R. K.A. Natale, et al. (1998). "Physiology of the escape rhythm after radiofrequency atrioventricular junctional ablation." Pacing Clin Electrophysiol 21(5): 1085-92.

Sherron, P.E. Torres-Arraut, et al. (1985). "Site of conduction delay and electrophysiologic significance of first-degree atrioventricular block in children with heart disease." Am J Cardiol 55(11): 1323-7.

Silka, M. and A. Garson Jr. (1999). Ventricular Arrhythmias. Clinical Pediatric Arrhythmias. P. Gillette and A. Garson Jr. Philadelphia, PA, WB Saunders: 121-145.

Silka, M. J. and A. Garson, Jr. (1999). Ventricular Arrhythmias. Clinical Pediatric Arrhythmias. P. C. Gillette and A. Garson, Jr. Philadelphia, W.B. Saunders Company: 121-145.

Skinner JR, Sharland G. Detection and management of life threatening arrhythmias in the perinatal period. Early Human Development 2008;84(3 ):161-172..

Sobotka, P. A.J. H. Mayer, et al. (1981). "Arrhythmias documented by 24-hour continuous ambulatory electrocardiographic monitoring in young women without apparent heart disease." Am Heart J 101(6): 753-9.

Sokoloski, M. (1999). Tachyarrhythmias Confined to the Atrium. Clinical Pediatric Arrhythmias. P. Gillette and A. Garson Jr. Philadelphia, PA, WB Saunders.

Southall, D. P.J. Richards, et al. (1980). "Study of cardiac rhythm in healthy newborn infants." Br Heart J 43(1): 14-20.

Stein, R.C. M. Medeiros, et al. (2002). "Intrinsic sinus and atrioventricular node electrophysiologic adaptations in endurance athletes." J Am Coll Cardiol 39(6): 1033-8.

Still, A.P. Raatikainen, et al. (2005). "Prevalence, Characteristic and Natural Course of Inappropriate Sinus Tachycardia." Europace 7: 104-12.

Strasberg, B.Y. L. F. Amat, et al. (1981). "Natural history of chronic second-degree atrioventricular nodal block." Circulation 63(5): 1043-9.

Sugimoto, T.H. Hayakawa, et al. (1986). "Clinical evaluation of bisoprolol in the treatment of extrasystoles and sinus tachycardia: an interim report." J Cardiovasc Pharmacol 8 Suppl 11: S171-4.

Talbot, S. and M. Greaves (1976). "Association of ventricular extrasystoles and ventricular tachycardia with idioventricular rhythm." Br Heart J 38(5): 457-64.

Texter, K.N. Kertesz, et al. (2006). "Atrial Flutter in Infants." Journal of The American College of Cardiology 48(5): 1040-6.

Triedman, J. (2001). Atrial Reentrant Tachycardias. Cardiac Arrhythmias in Children and Young Adults with Congenital Heart Disease. E. Walsh, J. Saulet al. Philadelphia, PA, Lippincott Williams \& Wilkins: 137-159.

Triedman, J. (2004). Atrial Arrhythmias in Congenital Heart Disease. Cardiac Electrophysiology From Cell to Bedside. D. Zipes and J. Jaliffe. Philadelphia, PA: 558568.

Triedman, J.D. Bergau, et al. (1997). "Efficacy of Radiofrequency Ablation for Control of Intraatrial Reentry Tachycardia in Patient with Congenital Heart Disease." Journal of The American College of Cardiology 30(4): 1032-38.

Vaksmann, G.C. D'Hoinne, et al. (2006). "Permanent junctional reciprocating tachycardia in children: a multicentre study on clinical profile and outcome." Heart 92(1): 101-4.

Vaksmann, G.A. Fournier, et al. (1990). "Frequency and prognosis of arrhythmias after operative "correction" of tetralogy of Fallot." Am J Cardiol 66(3): 346-9. 
Valimaki, I. and P. A. Tarlo (1971). "Heart rate patterns and apnea in newborn infants." Am J Obstet Gynecol 110(3): 343-9.

Van Hare, G. (1999). Supraventricular Tachycardia. Clinical Pediatric Arrhythmias. P. Gillette and A. Garson Jr. Philadelphia, W.B. Saunders Company: 97-120.

Villain, E.V. Vetter, et al. (1990). "Evolving Concepts in the Management of Congenital Junctional Ectopic Tachycardia. A Multicenter Study." Circulation 81(5): 1544-9.

von Bernuth, G.R. Toussaint, et al. (1989). "[Heart rate and heart rhythm in healthy infants and children]." Klin Padiatr 201(2): 98-103.

Waldo, A. (2004). Atrial Flutter: Mechanisms, Clinical Features, and Management. Cardiac Electrophysiology From Cell to Bedside. D. Zipes and J. Jaliffe. Philadelphia, PA, Saunders: 490-499.

Wallace, A. G. and W. M. Daggett (1964). "Re-Excitation of the Atrium. "the Echo Phenomenon"." Am Heart J 68: 661-6.

Walsh, E. (2001a). Automatic Atrial and Junctional Tachycardias. Cardiac Arrhythmias in Children and Young Adults with Congenital Heart Disease. E. Walsh, J. Saulet al. Philadelphia, PA, Lippincott Williams \& Wilkins: 115-135.

Walsh, E. (2001b). Diagnosis and Acute Management of Tachycardias. Cardiac Arrhythmias in Children and Young Adults with Congenital Heart Disease. E. Walsh, J. Saulet al. Philadelphia, Lippincott Williams \& Wilkins: 95-113.

Walsh, E. P. and F. Cecchin (2007). "Arrhythmias in adult patients with congenital heart disease." Circulation 115(4): 534-45.

Weindling, S. (2001). Atrioventricular Conduction Disturbances. Cardiac Arrhythmias in Children and Young Adults with Congenital Heart Disease. E. Walsh, J. Saulet al. Philadelphia, Lippincott Williams \& Wilkins: 285-300.

Wit, A. L. and P. F. Cranefield (1977). "Triggered and automatic activity in the canine coronary sinus." Circ Res 41(4): 434-45.

Yang, Y.J. Cheng, et al. (2001). "Atypical Right Atrial Flutters Patterns." Circulation 103(25): 3092-8.

Young, D.R. Eisenberg, et al. (1977). "Wenckebach atrioventricular block (Mobitz type I) in children and adolescents." Am J Cardiol 40(3): 393-9.

Zhu, D. W.J. D. Maloney, et al. (1995). "Radiofrequency catheter ablation for management of symptomatic ventricular ectopic activity." J Am Coll Cardiol 26(4): 843-9.

Zimmerman, F. (2001). Atrioventricular Node Reentry Tachycardia. Cardiac Arrhythmias in Children and Young Adults with Congenital Heart Disease. E. Walsh, J. Saulet al. Philadelphia, PA, Lippincott Williams \& Wilkins: 161-172.

Zipes, D. and A. Camm (2006). "ACC/AHA/ESC 2006 guidelines for management of patients with ventricular arrhythmias and the prevention of sudden cardiac death." Journal of The American College of Cardiology 48(5): e247-346.

Zipes, D. P.J. P. DiMarco, et al. (1995). "ACC/AHA Guidelines for clinical intracardiac electrophysiological and catheter ablation procedures. A report of the American College of Cardiology/American Heart Association Task Force on Practice Guidelines (Committee on Clinical Intracardiac Electrophysiologic and Catheter Ablation Procedures), developed in collaboration with the North American Society of Pacing and Electrophysiology." J Am Coll Cardiol 26(2): 555-73. 
Zipes, D. P. and A. Garson, Jr. (1994). "26th Bethesda conference: recommendations for determining eligibility for competition in athletes with cardiovascular abnormalities. Task Force 6: arrhythmias." J Am Coll Cardiol 24(4): 892-9. 


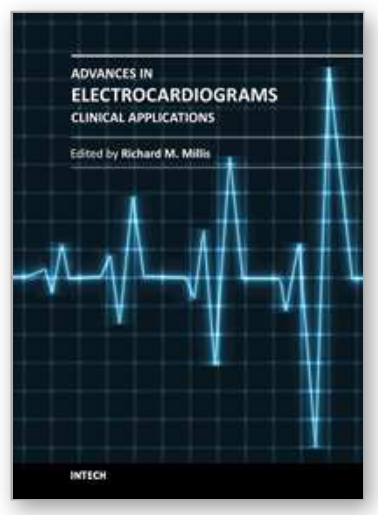

\author{
Advances in Electrocardiograms - Clinical Applications \\ Edited by PhD. Richard Millis
}

ISBN 978-953-307-902-8

Hard cover, 328 pages

Publisher InTech

Published online 25, January, 2012

Published in print edition January, 2012

Electrocardiograms have become one of the most important, and widely used medical tools for diagnosing diseases such as cardiac arrhythmias, conduction disorders, electrolyte imbalances, hypertension, coronary artery disease and myocardial infarction. This book reviews recent advancements in electrocardiography. The four sections of this volume, Cardiac Arrhythmias, Myocardial Infarction, Autonomic Dysregulation and Cardiotoxicology, provide comprehensive reviews of advancements in the clinical applications of electrocardiograms. This book is replete with diagrams, recordings, flow diagrams and algorithms which demonstrate the possible future direction for applying electrocardiography to evaluating the development and progression of cardiac diseases. The chapters in this book describe a number of unique features of electrocardiograms in adult and pediatric patient populations with predilections for cardiac arrhythmias and other electrical abnormalities associated with hypertension, coronary artery disease, myocardial infarction, sleep apnea syndromes, pericarditides, cardiomyopathies and cardiotoxicities, as well as innovative interpretations of electrocardiograms during exercise testing and electrical pacing.

\title{
How to reference
}

In order to correctly reference this scholarly work, feel free to copy and paste the following:

Harinder R. Singh (2012). Arrhythmias in Children and Young Adults, Advances in Electrocardiograms Clinical Applications, PhD. Richard Millis (Ed.), ISBN: 978-953-307-902-8, InTech, Available from: http://www.intechopen.com/books/advances-in-electrocardiograms-clinical-applications/arrhythmias-inchildren-and-young-adults

\section{INTECH}

open science | open minds

\section{InTech Europe}

University Campus STeP Ri

Slavka Krautzeka 83/A

51000 Rijeka, Croatia

Phone: +385 (51) 770447

Fax: +385 (51) 686166

www.intechopen.com

\section{InTech China}

Unit 405, Office Block, Hotel Equatorial Shanghai

No.65, Yan An Road (West), Shanghai, 200040, China 中国上海市延安西路65号上海国际贵都大饭店办公楼405单元

Phone: $+86-21-62489820$

Fax: +86-21-62489821 
(C) 2012 The Author(s). Licensee IntechOpen. This is an open access article distributed under the terms of the Creative Commons Attribution 3.0 License, which permits unrestricted use, distribution, and reproduction in any medium, provided the original work is properly cited. 